\title{
Impact of Higher Harmonics in Searching for Gravitational Waves from Non-Spinning Binary Black Holes
}

\author{
Collin Capano, ${ }^{1}$ Yi Pan, ${ }^{1}$ and Alessandra Buonanno ${ }^{1}$ \\ ${ }^{1}$ Maryland Center for Fundamental Physics \& Joint Space Science Institute, \\ Department of Physics, University of Maryland, College Park, 20742
}

\begin{abstract}
Current searches for gravitational waves from coalescing binary black holes (BBH) use templates that only include the dominant harmonic. In this study we use effective-one-body multipolar waveforms calibrated to numerical-relativity simulations to quantify the effect of neglecting sub-dominant harmonics on the sensitivity of searches. We consider both signal-to-noise ratio (SNR) and the signal-based vetoes that are used to re-weight SNR. We find that neglecting sub-dominant modes when searching for non-spinning BBHs with component masses $3 \mathrm{M}_{\odot} \leq m_{1}, m_{2} \leq 200 \mathrm{M}_{\odot}$ and total mass $M<360 \mathrm{M}_{\odot}$ in advanced LIGO results in a negligible reduction of the re-weighted SNR at detection thresholds. Sub-dominant modes therefore have no effect on the detection rates predicted for advanced LIGO. Furthermore, we find that if sub-dominant modes are included in templates the sensitivity of the search becomes worse if we use current search priors, due to an increase in false alarm probability. Templates would need to be weighted differently than what is currently done to compensate for the increase in false alarms. If we split the template bank such that sub-dominant modes are only used when $M \gtrsim 100 \mathrm{M}_{\odot}$ and mass ratio $q \gtrsim 4$, we find that the sensitivity does improve for these intermediate mass-ratio BBHs, but the sensitive volume associated with these systems is still small compared to equal-mass systems. Using sub-dominant modes is therefore unlikely to substantially increase the probability of detecting gravitational waves from non-spinning $\mathrm{BBH}$ signals unless there is a relatively large population of intermediate mass-ratio BBHs in the universe.
\end{abstract}

\section{INTRODUCTION}

Within the next few years the next generation of gravitational-wave detectors will come online. These detectors - the advanced Laser Interferometer Gravitational-wave Observatory (aLIGO) in the United States 1, the French-Italian Virgo observatory [2], the KAGRA detector in Japan 3, and a potential third LIGO detector in India [4 - will be sensitive to sources up to 10 times more distant than first generation detectors. One of the most promising sources of gravitationalwaves for these detectors are coalescing binary black holes (BBHs). As the two black holes in a binary orbit each other, they emit gravitational radiation; this causes them to inspiral and eventually merge into a single black hole. Advanced detectors will be able to detect radiation emitted during this process up to tens of Gpc away. The rate of $\mathrm{BBH}$ coalescences with masses detectable by the advanced detectors is highly uncertain: the detection rate has been estimated to be as low as $0.4 \mathrm{yr}^{-1}$, but it may be as high as $1000 \mathrm{yr}^{-1}$ [5]. If the optimistic end of this range is correct, BBHs would be the most prolific source of gravitational-wave detections in the advanced detector era.

Searches for BBHs use a matched filter to determine when a gravitational wave is likely to be present in a detector's data [6, 7], 1 . The filter produces a signal-tonoise ratio (SNR) that is proportional to the probability

\footnotetext{
${ }^{1}$ Un-modeled "burst" searches [8 are also sensitive to BBHs [9]. Previously, these searches have been used to search for signals with total masses $M>100 \mathrm{M}_{\odot}$, as the signals in this mass range did not have many cycles in the sensitive band of the
}

that a signal exists in the data with similar parameters as the template used in the correlation [10. Since the physical parameters of the signal are unknown a priori, a discrete bank of templates is used to cover the range of possible parameters [11, 12]. This technique for searching for gravitational waves relies on good agreement between templates and real signals. If there is significant disagreement between the two, the SNR will be reduced, and it becomes difficult to separate potential signals from noise. It is therefore important to verify that templates adequately resemble potential gravitational-wave signals.

Gravitational waveforms are decomposed into a -2 spin-weighted spherical harmonic basis ${ }_{2} Y_{l m}(\theta, \phi)$. Template waveforms used in past searches for $\mathrm{BBHs}$ have only included the most dominant mode, $l=|m|=2$ [6, 7]. In addition, predictions of the number of detections that will be made in advanced LIGO have been made assuming both templates and signals only have the dominant mode [5. Real signals, however, will have all modes. There can be significant mismatch [defined as $1-\mathcal{E}$, where $\mathcal{E}$ is given by Eq. 13 see Sec. IIC 1 for details] between waveforms that include sub-dominant modes and waveforms that only include the dominant mode 13 16. This raises the question, by neglecting sub-dominant modes has the sensitivity of BBH searches been overstated? Furthermore, would sensitivity improve if templates included sub-dominant modes?

initial generation of LIGO detectors. In this paper, we will consider signals with $M$ as large as $360 \mathrm{M}_{\odot}$. Due to the improved low-frequency $(<40 \mathrm{~Hz})$ sensitivity of the advanced detectors, template-based searches similar to those done in 6, 7 ] will be feasible at these higher masses. We therefore only consider template-based searches here. 
Several studies have investigated the effects of subdominant modes to try to answer these questions. Ref. [14] used waveforms generated from numerical relativity as both template and signal to measure mismatch when sub-dominant modes are not included in templates. They considered systems with total mass $M>100 \mathrm{M}_{\odot}$ and mass ratios $1 \leq q \leq 4 \mathrm{~L}^{2}$ When computing overlaps they only considered templates which had the same intrinsic parameters (mass and spin) as signals. They found that adding sub-dominant modes could improve detection volume by up to $30 \%$, but they noted that the largest gain in volume occurred for signals for which the detectors had the least sensitivity (these were systems with asymmetric masses and inclination angle - the angle between the orbital angular momentum and the line of sight to the detector $-\theta \approx \pi / 2$ ).

To fully understand the effect of sub-dominant modes on the sensitivity of $\mathrm{BBH}$ searches, a bank of template waveforms covering the source parameter space is necessary. These waveforms should contain the complete inspiral, merger and ringodwn stages of sub-dominant modes since the latter become relatively stronger during the last stage of inspiral and merger. Here we employ effective-one-body (EOB) waveforms [17, 18, calibrated to numerical-relativity simulations [13].

Building on an initial attempt 19 to calibrate subdominant modes, Ref. 13 built a nonspinning EOB model that include four sub-dominant modes, namely the $(l, m)=(2,1),(3,3),(4,4)$ and $(5,5)$ modes, as well as the dominant $(l, m)=(2,2)$ mode. The EOB model of Ref. [13] was calibrated to numerical-relativity simulations of mass ratios $q \leq 6.3$ A direct study [21] carried out with the Markov Chain Monte Carlo technique demonstrated that the EOB waveforms of Ref. [13] are indistinguishable from the numerical-relativity waveforms 22] used to calibrate them up to $\mathrm{SNR}=50$ for the advanced LIGO detectors. Furthermore, preliminary studies in Ref. 13] suggested that the EOB waveforms containing subdominat modes could be sufficiently accurate to search for nonspinning $\mathrm{BBH}$ signals of $q \leq 6$, at least in the relatively narrow frequency band where direct comparison with numerical-relativity waveforms was possible. The recent investigation of Ref. 23] verified the accuracy of these EOB waveforms in the entire sensitivity band of advanced LIGO detectors. Having the correct limit for $q \rightarrow \infty$ by construction, we expect EOB waveforms to be reasonably accurate when $q>6$. This expectation was recently reinforced by the excellent agreement found against a $q=10$ numerical-relativity waveform 24. Finally, the dominant mode EOB waveforms that we use here have been employed as simulated signals in the most recent LIGO BBH searches [7].

\footnotetext{
${ }^{2}$ In this paper we use the convention that $q=m_{1} / m_{2}$ with $m_{1} \geq$ $m_{2}$.

3 An EOB model with slightly different parametrization was calibrated to the same set of numerical-relativity waveforms and provides two sub-dominant modes $(l, m)=(2,1)$ and $(3,3)$ [20].
}

Using a bank of dominant-mode templates that allows maximization over the masses of the binaries, Ref. [15] studied templates and signals with component masses $3 \mathrm{M}_{\odot} \leq m_{1}, m_{2} \leq 25 \mathrm{M}_{\odot}$. They also found that subdominant modes had little effect on equal-mass systems, but argued that sensitive volume could be increased by as much as $25 \%$ for systems with $q \geq 4$ and inclination angles $1.08 \mathrm{rad} \leq \theta \leq 2.02 \mathrm{rad}$ if sub-dominant modes were added to templates.

Both of these studies calculated what percentage of sensitive volume could be gained if sub-dominant modes were added to templates by finding the fractional loss in SNR of signals with sub-dominant modes when they were recovered by dominant-mode templates. However, estimating the gain in sensitive volume in this manner neglects additional complications that arise when searching in real, non-Gaussian, data. In real data a signalbased veto, $\chi^{2}$ 25, is used to re-weight SNR [6, 7]. Reweighted $S N R$ is needed in order to separate potential gravitational-wave signals from non-Gaussian transients that are present in detector data [26]. Any mismatch between the templates and signals causes an increase in $\chi^{2}$, which in turn causes a decrease in re-weighted SNR relative to SNR. Thus the sensitivity of dominant-mode templates to real signals may be worse than predicted from SNR considerations alone. This makes the case for adding sub-dominant modes to templates stronger.

A signal must have high statistical significance in order for it to be considered a gravitational-wave candidate. The standard measurement of significance is falsealarm probability, which is the probability that an event caused by noise is mis-identified as a signal. When calculating sensitive volume, the SNR threshold is chosen such that the false-alarm probability at that threshold is small. In Refs. [14] and [15] the same SNR threshold was used when estimating the sensitive volume of dominantmode templates and the potential sensitive volume of sub-dominant mode templates. Both studies acknowledged that adding sub-dominant modes to templates can increase the probability of getting a false alarm. To keep the false-alarm probability fixed, the SNR threshold in a search that uses sub-dominant mode templates must therefore increase. This increase in threshold decreases the sensitive volume that can be obtained by subdominant mode templates, making the case for adding sub-dominant modes to templates weaker.

Due to these conflicting factors it is difficult to make a definitive statement about the impact of sub-dominant modes on BBH searches from SNR considerations alone. In this paper we resolve the uncertainty by finding both SNR and $\chi^{2}$ between templates without sub-dominant modes and signals with sub-dominant modes. Doing so, we are able to estimate the fraction of sensitive volume that is lost by neglecting sub-dominant modes when reweighted SNR is used. We also simulate a search with sub-dominant modes: we estimate the increase in threshold needed to keep the false-alarm probability fixed, thereby allowing an accurate comparison of search vol- 
umes when sub-dominant modes are included and excluded in templates. We consider non-spinning BBHs with component-masses $3 \mathrm{M}_{\odot} \leq m_{1}, m_{2} \leq 200 \mathrm{M}_{\odot}$ and with total mass $M<360 \mathrm{M}_{\odot}$. We therefore cover the entire range of "stellar-mass" BBHs that were searched for in LIGO and Virgo data in the past $\left(m_{1}, m_{2} \in\right.$ $\left.[3,97] \mathrm{M}_{\odot} ; M \leq 100 \mathrm{M}_{\odot}\right)$ [7, 27], and we cover binaries that involve "intermediate-mass black holes" which may form from dynamical capture in globular clusters [28]. To generate both dominant-mode and subdominant mode waveforms we use the EOB model calibrated to numerical-relativity simulations, as obtained in Ref. [13].

For simplicity, and as was done in Refs. [14, 15, we study the effect of sub-dominant modes using a single detector (real searches use a network of detectors). We simulate an advanced LIGO detector by generating stationary Gaussian noise colored by the zero-detuned, highpower advanced LIGO design curve [29]. Due to the presence of non-Gaussian transients there is currently no model for the noise distribution of real detector data [30, 31. However, by injecting signals into Gaussian noise we find the best sensitivity that can be obtained by the search. We reason that if sub-dominant mode templates have worse sensitivity than dominant-mode templates in Gaussian noise, those templates will fare no better in real detector data. Furthermore, current detection pipelines are able to mitigate noise transients such that the sensitive volumes of real detectors are within a factor of a few of what they would be if the data were Gaussian [26. The sensitive volumes we find using Gaussian noise is thus a good approximation of what they will be in real detector data (assuming advanced detectors have similar data-quality characteristics).

In this paper we are concerned primarily with the effects of sub-dominant modes on our ability to detect gravitational waves; we do not address the effect on parameter estimation. The goal of detection is to determine whether or not a signal exists in some data, regardless of the parameters. When doing parameter estimation, on the other hand, a signal is assumed to be in the data; the goal is then to find the best fitting parameters 4 These differing goals put different constraints on what to use as template waveforms. As we will see, including sub-dominant modes in templates does not improve our ability to detect if the sub-dominant modes do not increase the SNR of signals enough to offset increases in false-alarm probability. If a signal is assumed to be present, however, then adding sub-dominant modes to template waveforms can only improve parameter estimation if the resulting waveform is a better match to the signal. Indeed, it has been shown [16, 21] that including sub-dominant modes reduces systematic bias when measuring the parameters

\footnotetext{
${ }^{4}$ In prior searches, a detection pipeline was run on data first to identify times when candidate signals exist; these times were then followed up by parameter estimation pipelines [32.
}

of signals.

The rest of this paper is divided as follows: Sec. II provides background for the search methods and statistics we discuss. In Sec. IIA we review how SNR is calculated using dominant-mode templates; in Sec. IIB we review the $\chi^{2}$ statistic and how it is used to re-weight the SNR; in Sec. IIC we discuss the statistics we use in this paper to compare the sensitivity of searches; in Sec. IID we provide a brief review of the astrophysics of BBHs to motivate our choice of masses and rate priors. We show that the bank of dominant-mode templates we use is effectual to dominant-mode signals across the mass space we investigate in Sec. IIIA. In Sec. IIIB we find the sensitivity of this bank to signals with sub-dominant modes to see if sub-dominant modes have an effect on predicted sensitivity. In Sec. IV we estimate the sensitivity of a simulated bank of sub-dominant mode templates using equations derived in Appendices B and A Finally, in Sec. $\mathrm{V}$ we review and discuss our results.

\section{REVIEW OF CURRENT SEARCHES AND TEMPLATE MODELING}

The strain induced in a detector from a passing gravitational wave is [33]:

$$
\begin{aligned}
h(t ; \mathbf{\Upsilon}, \boldsymbol{\Xi})=\frac{1}{r} & \left(F_{+}(\alpha, \delta, \psi) h_{+}\left(t-t_{c} ; \theta, \phi, \phi_{0}, \mathbf{\Upsilon}\right)\right. \\
& \left.+F_{\times}(\alpha, \delta, \psi) h_{\times}\left(t-t_{c} ; \theta, \phi, \phi_{0}, \mathbf{\Upsilon}\right)\right)
\end{aligned}
$$

where:

$$
\begin{aligned}
& h_{(+, \times)}=(\Re, \Im) \sum_{l m}{ }_{-2} Y_{l m}(\theta, \phi) A_{l m}\left(t-t_{c} ; \mathbf{\Upsilon}\right)
\end{aligned}
$$

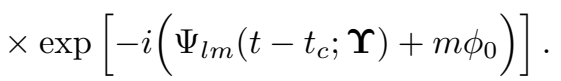

Here, ${ }_{-2} Y_{l m}(\theta, \phi)$ are the -2 spin-weighted spherical harmonics, $r$ is the distance to the source from the detector, $\phi_{0}$ is the initial phase of the binary, and $t_{c}$ is the coalescence time of the binary. The angle $\theta$ is the angle between the orbital-angular momentum and the line-ofsight to the detector (the inclination); $\phi$ is the azimuthal angle to the projection of the line-of-sight on to the orbital plane. The functions $F_{+}$and $F_{\times}$project the gravitational wave from the source's radiation frame into the detector's frame; they are functions of the right ascension $(\alpha)$, declination $(\delta)$, and polarization $(\psi)$ of the source with respect to the detector 34.5 Since we only consider non-spinning waveforms in this paper, the intrinsic parameters $\Upsilon$ are the component masses $m_{1}$ and $m_{2}$. Neglecting spin also means that $\phi$ and $\phi_{0}$ are degenerate, and we can set $\phi_{0}=0$. Together the parameters $\left\{t_{c}, r, \theta, \phi, \alpha, \delta, \psi\right\}$ make up the extrinsic parameters $\boldsymbol{\Xi}$.

\footnotetext{
${ }^{5}$ Due to the motion of the Earth, $F_{+}$and $F_{\times}$are also functions of time. Here we assume that the relative displacement of the detector is small across the duration of the signal in the detector's band.
} 


\section{A. SNR maximization for a dominant mode bank}

Given some detector data $s$ we wish to determine whether or not a gravitational-wave signal $h$ is present in it. We do not know a priori the parameters of $h$. In order to maximize the probability of detection we must therefore search over all possible intrinsic and extrinsic parameters that $h$ may have. To do this, we calculate the signal-to-noise (SNR) $\rho$ maximized over $\boldsymbol{\Upsilon}$ and $\boldsymbol{\Xi}$ :

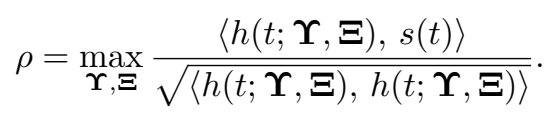

The inner product $\langle\cdot, \cdot\rangle$ is defined as [35]:

$$
\langle a, b\rangle=4 \Re \int_{0}^{\infty} \frac{\tilde{a}^{*}(f) \tilde{b}(f)}{S_{n}(f)} \mathrm{d} f,
$$

where $S_{n}(f)$ is the one-sided power spectral density (PSD) of the noise. In this paper we use the zerodetuned, high-power, advanced LIGO design curve [29]. This PSD grows substantially at frequencies below $10 \mathrm{~Hz}$ due to seismic noise. As was done in Ref. [15], we use a lower frequency cutoff of $15 \mathrm{~Hz}$ for our matched filter. We terminate the filter at a frequency larger than the ringdown frequency of the signal.

In principle we must maximize the SNR over 8 parameters for non-spinning systems -2 intrinsic plus 6 extrinsid _ but this number can be reduced. Let:

$$
\begin{aligned}
|F| & =\sqrt{F_{+}^{2}+F_{\times}^{2}}, \\
\kappa & =\arctan \left(\frac{F_{\times}}{F_{+}}\right) ;
\end{aligned}
$$

then:

$$
h=\frac{1}{\mathcal{D}}\left(h_{+} \cos \kappa+h_{\times} \sin \kappa\right),
$$

where $\mathcal{D}=|F| / r$ is the effective distance 35. Since $\mathcal{D}$ cancels in the SNR, we need not maximize over it. We can thus maximize over $\alpha, \delta$, and $\psi$ by simply maximizing $\kappa$.

The coalescence time is maximized over by evaluating the SNR at discrete time intervals, selecting points where $\rho$ is at a maximum and exceeds some threshold. These points are triggers. In Ref. [35] it is shown that $\rho(t)$ can be efficiently calculated by taking the inverse Fourier transform of $\tilde{h}^{*}(f) \tilde{s}(f) / S_{n}(f)$. Since we are interested in the effect of sub-dominant modes, which do not affect the maximization over coalescence time, we will make this maximization implicit and set $t_{c}=0$ throughout the rest of this paper.

In order to maximize over the intrinsic parameters a template bank is used [11, 12]. Templates are typically

\footnotetext{
${ }^{6}$ We do not need to maximize over $r$ since it cancels in the SNR.
}

laid out across the search parameter space such that no more than $3 \%$ of the SNR is lost due to the discreetness of the bank [6, 7]. The SNR is maximized over the extrinsic parameters for each trigger; the template with the largest SNR is then selected, thereby maximizing over the intrinsic parameters.

With these considerations Eq. (3) simplifies to:

$$
\rho=\max _{\theta, \phi, \kappa} \frac{\left\langle h_{+}(t ; \theta, \phi), s(t)\right\rangle \cos \kappa+\left\langle h_{\times}(t ; \theta, \phi), s(t)\right\rangle \sin \kappa}{\sqrt{\langle h(t ; \kappa, \theta, \phi), h(t ; \kappa, \theta, \phi)\rangle}} .
$$

If the templates contain sub-dominant modes, then the number of parameters that need to be maximized over can be reduced no further. In Appendix A we perform the maximization over $\kappa$ analytically when sub-dominant modes are included (the maximization over $\theta$ and $\phi$ must be done numerically). This is used to model a hypothetical search using sub-dominant modes, which is discussed in Sec. IV In current searches, template waveforms are generated using only the dominant, $l=|m|=2$, mode. In that case $\theta, \phi$, and $\kappa$ are all degenerate with each other and the maximization reduces to a single parameter. This can be performed analytically, yielding [10:

$$
\rho=\sqrt{\frac{\left\langle h_{+}(t), s(t)\right\rangle^{2}+\left\langle h_{\times}(t), s(t)\right\rangle^{2}}{\langle h, h\rangle}} .
$$

In stationary Gaussian noise containing no signal, $\rho$ is $\chi$ distributed (or equivalently, $\rho^{2}$ is $\chi^{2}$ distributed) with two degrees of freedom.

\section{B. The $\chi^{2}$ test and re-weighted SNR}

Real data from the LIGO and Virgo detectors contain a number of non-Gaussian transients (glitches) [30, 31]. To mitigate the effect of these glitches the $\chi^{2}$ statistic is calculated to better separate noise from potential signals. This is calculated as follows: split the matched filter into $p$ frequency bins such that the template has equal amounts of power in each bin, and let $\rho_{i}$ be the SNR of a trigger in the $i^{\text {th }}$ bin. If the signal matches the template then $\rho_{i} \approx \rho / p$. We can therefore quantify how well a signal matches a template by defining [25]:

$$
\chi^{2}=p \sum_{i=1}^{p}\left|\rho_{i}-\frac{\rho}{p}\right|^{2}
$$

If the template matches the signal exactly then the $\chi^{2}$ statistic will be $\chi^{2}$ distributed with $2 p-2$ degrees of freedom (hence the name). If there is any mismatch between the signal and the template, the mean $\chi^{2}$ is [25]:

$$
\left\langle\chi^{2}\right\rangle=2 p-2+\mu^{2} \rho^{2}
$$

where $\mu$ is a measure of the mismatch. Thus the larger the mismatch between the signal and the template, the 
larger the $\chi^{2}$ statistic. Since the increase in $\chi^{2}$ is proportional to $\rho$, even "loud" (large SNR) glitches are mitigated by $\chi^{2}$ as they will have high mismatch to the template.

In $\mathrm{BBH}$ searches $\chi^{2}$ is used to re-weight $\rho$, obtaining the re-weighted $S N R \tilde{\rho}]^{7}$ As it is based on characteristics of the data and the templates, the exact form of the weighting evolved throughout initial LIGO. Generally, the weighting is such that $\rho$ is reduced for triggers with high $\chi^{2}$. In this study we use the re-weighted SNR that was used in the most recent searches [6, 7, which is defined as 8

$$
\tilde{\rho}=\left\{\begin{array}{cc}
\rho & \text { for } \chi_{r}^{2} \leq 1, \\
\rho\left[\frac{1}{2}\left(1+\left(\chi_{r}^{2}\right)^{3}\right)\right]^{-1 / 6} & \text { for } \chi_{r}^{2}>1 .
\end{array}\right.
$$

where $\chi_{r}^{2}$ is the reduced $\chi^{2}$, which is $\chi^{2}$ divided by the number of degrees of freedom.

Re-weighting the SNR using $\chi^{2}$ is crucial to $\mathrm{BBH}$ searches. An SNR of 8 is typically used when predicting detection rates $[5$, but it would not be possible to detect at this $\rho$ without some type of $\chi^{2}$ re-weighting of glitches [26]. However, if signals also significantly mismatch the templates then the use of $\tilde{\rho}$ can adversely affect the efficiency to these signals. The effect on efficiency from the mismatch between signals and templates therefore cannot be determined from the loss in SNR alone. For this reason, in the following sections we investigate the effect on $\chi^{2}$ due to the mismatch between dominant-mode templates and signals with sub-dominant modes, and we calculate efficiency using $\tilde{\rho}$.

\section{Quantifying the ability of a search to make detections}

The purpose of our study is to investigate whether adding sub-dominant modes to templates will improve our ability to detect gravitational waves from nonspinning BBHs. Ultimately, we want to know what template bank maximizes the number of detections made per unit time. To do so we need to quantify the ability of a bank with dominant-mode templates and a bank of templates with sub-dominant modes to recover waveforms with sub-dominant modes. For this purpose we calculate effectualness; we also calculate efficiency, from which we find sensitive volume and relative gain.

\footnotetext{
${ }^{7}$ A cut on large $\chi^{2}$ values is also employed, but this cut is chosen conservatively in order to not remove any signals [26]. As such, it is mostly used to reduce the number of triggers for computational purposes. We therefore do not consider it here.

${ }^{8}$ In Ref. 7, a different weight than that given in Eq. 12 was used for triggers whose templates had duration $<0.2 \mathrm{~s}$. All of the templates in our study have durations $>1 \mathrm{~s}$, however, due to the better low-frequency performance of the advanced LIGO noise curve we use. We therefore use Eq. 12 for all triggers.
}

\section{Effectualness}

Effectualnes $\AA^{9}$ is a statistic that is commonly used to quantify how well one family of waveforms recovers another. Given a simulated signal $h^{\dagger}$ with parameters $\boldsymbol{\Theta}=(\boldsymbol{\Upsilon}, \boldsymbol{\Xi})$ and a bank of templates $\left\{h\left(\boldsymbol{\Theta}^{\prime}\right)\right\}$, effectualness is defined as [36, 37]:

$$
\mathcal{E}_{a b}(\boldsymbol{\Theta})=\max _{\boldsymbol{\Theta}^{\prime}} \frac{\left\langle h_{a}\left(\boldsymbol{\Theta}^{\prime}\right), h_{b}^{\dagger}(\boldsymbol{\Theta})\right\rangle}{\sqrt{\left\langle h_{a}\left(\boldsymbol{\Theta}^{\prime}\right), h_{a}\left(\boldsymbol{\Theta}^{\prime}\right)\right\rangle\left\langle h_{b}^{\dagger}(\boldsymbol{\Theta}), h_{b}^{\dagger}(\boldsymbol{\Theta})\right\rangle}} .
$$

Here and elsewhere we adopt subscripts on $\mathcal{E}$ to indicate whether or not the signal and templates have subdominant modes; the first index indicates the templates, the second the signal. We will use $\mathrm{S}$ to indicate a waveform that has sub-dominant modes and $\mathrm{D}$ to indicate a waveform without sub-dominant modes. For example, $\mathcal{E}_{\mathrm{DS}}(\boldsymbol{\Theta})$ is the effectualness of a bank of templates without sub-dominant modes to a signal with sub-dominant modes and parameters $\boldsymbol{\Theta}$.

If a signal $h^{\dagger}$ is in stationary Gaussian noise with zeromean, the expectation value of the overlap with a template $h$ is $\left\langle h, s=h^{\dagger}+n\right\rangle=\left\langle h, h^{\dagger}\right\rangle$. The maximum overlap occurs when $h$ and $h^{\dagger}$ have the same parameters and come from the same waveform model. Thus, via Eq. (3), the expectation value of the maximum recoverable SNR of a signal $h^{\dagger}$ is:

$$
\max \langle\rho\rangle=\sqrt{\left\langle h^{\dagger}, h^{\dagger}\right\rangle} .
$$

Effectualness therefore gives the fraction of available SNR in a signal $h_{b}^{\dagger}$ that is recovered by the template $h_{a}$ :

$$
\mathcal{E}_{a b}(\boldsymbol{\Theta})=\frac{\left\langle\rho_{a b}(\boldsymbol{\Theta})\right\rangle}{\max \left\langle\rho_{b b}(\boldsymbol{\Theta})\right\rangle} .
$$

Here, $\left\langle\rho_{a b}(\boldsymbol{\Theta})\right\rangle$ indicates the expectation value of the SNR of signal $h_{b}^{\dagger}(\boldsymbol{\Theta})$ using templates $\left\{h_{a}\left(\boldsymbol{\Theta}^{\prime}\right)\right\}{ }^{10}$ The smaller the effectualness, the less SNR recovered by the template and the closer signals need to be in order to detect them. Effectualness is thus an estimate of how sensitive a bank of templates will be to a particular set of signals.

However, effectualness is not sufficient for comparing the sensitivity of a set of templates to signals created with different waveform models. We see from Eq. 115 that a drop in effectualness can result from a decrease in the overlap between two waveforms or from an increase in the

\footnotetext{
${ }^{9}$ Effectualness has also been referred to as "fitting factor" 36].

${ }^{10}$ Note that $\max \left\langle\rho_{b b}(\boldsymbol{\Theta})\right\rangle$ is not the same as $\left\langle\rho_{b b}(\boldsymbol{\Theta})\right\rangle$. This is because $\left\langle\rho_{b b}(\boldsymbol{\Theta})\right\rangle$ indicates the SNR of a bank of templates $\left\{h_{b}\left(\boldsymbol{\Theta}^{\prime}\right)\right\}$ to a signal $h_{b}^{\dagger}(\boldsymbol{\Theta})$. In fact, while the waveform models may be the same, the template and signal parameters may not due to the discreetness of the bank.
} 
maximum recoverable SNR. This ambiguity has particular relevance for sub-dominant modes. The predicted detection rates of advanced LIGO are made assuming that both signals and templates have the dominant mode only [5]. Real signals will have sub-dominant modes. If $\mathcal{E}_{\mathrm{DS}}<\mathcal{E}_{\mathrm{DD}}$ for some signals, it is not clear from effectualness alone whether the loss is due to $\left\langle\rho_{\mathrm{DS}}\right\rangle\left\langle\left\langle\rho_{\mathrm{DD}}\right\rangle\right.$ or if it is because $\max \left\langle\rho_{\mathrm{SS}}\right\rangle>\max \left\langle\rho_{\mathrm{DD}}\right\rangle$. If entirely the first case, detection rates will be less than predicted. If entirely the second case, the sensitivity of aLIGO will be the same as predicted (perhaps better, since the additional power in the sub-dominant modes may give larger SNR than expected). The drop in effectualness in this case only indicates that the sensitivity could be better than predicted if sub-dominant modes were added to templates, but it will not be worse.

It is also not clear what affect a decrease in effectualness has on re-weighted SNR. Equation (11) indicates that larger mismatch between signal and template results in larger $\chi^{2}$; this increase results in lower $\tilde{\rho}$. Since re-weighted SNR is used as the ranking statistic in real searches, the decrease in effectualness (increase in mismatch) may result in a further reduction in sensitivity via $\chi^{2}$.

Finally, effectualness implicitly assumes that SNR is the optimal statistic (in the Neyman-Pearson sense) to detect a gravitational wave in Gaussian noise. While this has been shown to be true when the intrinsic parameters of the signal are known 10, it is not necessarily true if the intrinsic parameters are unknown, as is the case in real $\mathrm{BBH}$ searches. Finding the optimal statistic in this case is difficult to do, as the waveforms have a non-trivial dependence on the intrinsic parameters. Moreover, we would need to know the distribution of the sources' intrinsic parameters, which in our case are the masses of BBHs. As we will see in Sec. IID the mass distribution of BBHs is highly uncertain. Even if we could find the optimal statistic for an assumed distribution, the statistic may not be optimal for the real astrophysical distribution.

In practice, we get around these difficulties by simply assuming that SNR maximized over the template bank is a good approximation to the optimal statistic. In doing so, we assume that each template is equally likely to detect a signal, which implicitly assumes a particular astrophysical distribution. Effectualness then gives us a measure of the performance of the bank assuming that distribution is correct. However, if the implicit distribution is not correct, then SNR maximized over the bank is not a good approximation of the optimal statistic, thereby making effectualness a poor metric for search sensitivity.

For these reasons the effect of sub-dominant modes on the sensitivity of a search cannot be ascertained by effectualness alone. A more informative metric is the sensitive volume, which is found from efficiency.

\section{Efficiency, sensitive volume, and relative gain}

Given a set of $N$ simulated signals with intrinsic and extrinsic parameters $\Theta$ and generated from waveformmodel $b,\left\{h_{b}^{\dagger}(\boldsymbol{\Theta})\right\}$, the efficiency of a bank of templates generated from waveform-model $a$ is the fraction of signals found with $\tilde{\rho}$ larger than some threshold value at a distance $r$ [38]; i.e.,

$$
\epsilon_{a b}(r, \mathbf{\Upsilon})=\frac{n_{a b}(r, \mathbf{\Upsilon})}{N_{b}(r, \mathbf{\Upsilon})}
$$

As with effectualness subscripts indicate the type of waveform used for the templates and signals; e.g., $\epsilon_{\mathrm{DS}}$ is the efficiency of a dominant mode bank to sub-dominant mode signals. Note that, aside from $r, \epsilon_{a b}$ is a function of the intrinsic parameters only $(\boldsymbol{\Upsilon})$. Since the universe is isotropic at the distances we are considering, the rest of the extrinsic parameters are accounted for by uniformly distributing signals in $\left\{\theta, \phi, \phi_{0}, \alpha, \delta, \psi\right\}$.

When determining efficiency in real searches the threshold $\tilde{\rho}$ is determined by the loudest event in the data [6, 7]. In this study we use $\tilde{\rho}=8$ as the threshold. SNR $(\rho)$ equal to 8 is commonly used as a threshold for predicting detection rates [5]. For well-matched signals, $\tilde{\rho} \approx \rho$ when $\rho=8$; using $\tilde{\rho}=8$ as a threshold should therefore give roughly the same results as predictions if mismatches between signals and templates are not too large.

Integrating the efficiency times the astrophysical rate of BBHs $R(V, \mathbf{\Upsilon})$ over volume and $\mathbf{\Upsilon}$ gives the expected rate of $\mathrm{GW}$ detections per unit time:

$$
\mathcal{R}_{\text {detect }}=\int \epsilon(r, \mathbf{\Upsilon}) R(V, \mathbf{\Upsilon}) \mathrm{d} V \mathrm{~d} \mathbf{\Upsilon}
$$

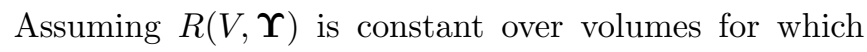
$\epsilon \neq 0$ and uniform in a small region in parameter space $\Upsilon+\Delta \Upsilon$, we define the sensitive volume $V_{a b}$ as:

$$
V_{a b}(\boldsymbol{\Upsilon}+\Delta \mathbf{\Upsilon})=4 \pi \int_{0}^{\infty} \epsilon_{a b}(r, \boldsymbol{\Upsilon}+\Delta \mathbf{\Upsilon}) r^{2} \mathrm{~d} r
$$

With these assumptions, $V_{a b}$ is proportional to the average rate of detections in $\boldsymbol{\Upsilon}+\Delta \mathbf{\Upsilon}$.

We assume that all of the error in the sensitive volume is due to error in the measurement of the efficiency from statistical fluctuations in the noise. This error is derived from the range of efficiency values for which the number of found injections varies no more than one standard deviation 39]:

$$
(n-\langle n\rangle)^{2} \leq\left\langle n^{2}\right\rangle-\langle n\rangle^{2} .
$$

The mean and variance are given by the Binomial distribution:

$$
\begin{aligned}
\langle n\rangle & =N \epsilon, \\
\left\langle n^{2}\right\rangle & =N \epsilon(1-\epsilon) .
\end{aligned}
$$


Using these values and solving for $\epsilon$ yields [39]:

$$
\pm \delta \epsilon_{i}=\frac{N_{i}\left(2 n_{i}+1\right) \pm \sqrt{4 N_{i} n_{i}\left(N_{i}-n_{i}\right)+N_{i}^{2}}}{2 N_{i}\left(N_{i}+1\right)}
$$

where the index $i$ indicates the efficiency and number of injections in the $i^{\text {th }}$ distance bin (dependence on waveforms $a, b$ and parameters $\boldsymbol{\Upsilon}$ made implicit). This error is then propagated to the volume via Eq. (18). Note that this results in a conservative estimate of $\delta V$. In our study we average over several realizations of noise when finding $\tilde{\rho}$; thus actual statistical fluctuations in $\epsilon_{a b}$ are less than what is assumed in Eq. (19).

To compare the sensitivity of two template banks to a set of simulated signals we define the relative gain $\mathcal{G}_{a b}^{c d}$ :

$$
\mathcal{G}_{a b}^{c d}(\boldsymbol{\Upsilon}+\Delta \mathbf{\Upsilon})=\frac{V_{c d}(\mathbf{\Upsilon}+\Delta \mathbf{\Upsilon})}{V_{a b}(\mathbf{\Upsilon}+\Delta \mathbf{\Upsilon})}
$$

The upper (lower) indices denote the waveform models used for templates and signals in the numerator (denominator). Since sensitive volume is proportional to the detection rate, the relative gain gives the average number of detections per unit time the $c d$ search will make relative to the $a b$ search.

\section{Astrophysical priors on the distribution of $\mathrm{BBH}$ masses}

When comparing the sensitivity of template banks it can happen that one bank has better sensitivity in one area of parameter space, but worse sensitivity in another. We will see this in Sec. IV templates with sub-dominant modes have better sensitivity at $M>100 \mathrm{M}_{\odot}$ and $q \gtrsim$ 10 , but worse sensitivity for lower mass and more equalmass systems. This raises the question of whether the gain in sensitivity in one area of parameter space is large enough to offset the loss in another. In this case it is useful to try to find the net relative gain, which is:

$$
\text { net }\left\{\mathcal{G}_{a b}^{c d}\right\}=\frac{\int R(\mathbf{\Upsilon}) V_{c d}(\mathbf{\Upsilon}) \mathrm{d} \mathbf{\Upsilon}}{\int R(\mathbf{\Upsilon}) V_{a b}(\mathbf{\Upsilon}) \mathrm{d} \mathbf{\Upsilon}} .
$$

Doing so gives the relative number of detections made over the entire parameter space; if the net gain is $>1$ the gain in sensitivity in one area of parameter space is worth the loss in another.

Calculating net gain requires knowledge of the rate of BBH coalescence, $R(\boldsymbol{\Upsilon})$. Unfortunately, this is highly uncertain. For a BBH with $m_{1}=m_{2}=10 \mathrm{M}_{\odot}$, the coalescence rate has been estimated to be anywhere from $O\left(10^{-4}\right)$ to $O\left(10^{-1}\right) \mathrm{Mpc}^{-3} \mathrm{Myr}^{-1}$, leading to predicted detection rates between 0.4 and $10^{3}$ per year in advanced LIGO [5]. We do not need to know the magnitude of the $\mathrm{BBH}$ coalescence rate in order to calculate the net gain - we only need the relative distribution in mass - but even this is largely uncertain.

Binary black holes with masses detectable by LIGO are thought to be formed in one of two ways: via the two stars in an isolated binary each collapsing into black holes (field binaries), or by a black hole capturing another black hole in a dense stellar region such as globular clusters [40. No BBHs have been directly observed; their existence is predicted from population synthesis models, from the predicted evolution of known X-ray binaries, and from considerations of the dynamics of black holes in dense stellar clusters [5].

The most massive black holes formed from stellar collapse known are in the X-ray binaries IC $10 \mathrm{X}-1$ and NGC 300 X-1 41, 42. These black holes have been estimated to have masses between 20 and $35 \mathrm{M}_{\odot}$, but population synthesis models predict that black holes formed from isolated field stars may have masses as large as $80 \mathrm{M}_{\odot}$ in low-metallicity environments [43. The mass distribution of black holes in field binaries are more difficult to predict, however, as the proximity of the two progenitor stars to each other add several complications [44]. Population synthesis models give varying results depending on the values assumed for input parameters, but models generally suggest field binaries have $M \lesssim 100 \mathrm{M}_{\odot}$ [45], with the peak of the distribution occurring around $20 \mathrm{M}_{\odot}$ (cf. Figs. 8 and 9 of Ref. 45). The distribution in mass ratio is also uncertain: some models predict equal-mass systems are more likely, while others predict a roughly uniform distribution between $q=1$ and 4 (cf. Fig. 9 of Ref. [44]).

Black holes formed from dynamical capture in globular clusters may have masses between $10^{2}$ and $10^{4} \mathrm{M}_{\odot}$ 28. The existence of these intermediate-mass black holes (IMBHs) is more speculative, but are supported by observations of ultra luminous X-ray sources [46]. If IMBHs do exist they may form binaries with other IMBHs or with stellar-mass black holes, thus forming BBHs with larger total masses and mass ratios than possible in field binaries [5]. The merger rate between an IMBH with mass between 50 and $350 \mathrm{M}_{\odot}$ and a stellarmass black hole with mass $m$ has been estimated to be $\sim(0.02 / m) \mathrm{Mpc}^{-3} \mathrm{Myr}^{-1}$ [4]. This is an optimistic estimate [5], but if correct, the rate of these intermediatemass-ratio inspirals (IMRIs) could be on the same order of magnitude as BBHs formed from field binaries.

In this paper we consider BBHs with $3 \leq m_{1}, m_{2} \leq$ $200 \mathrm{M}_{\odot}$ and $M \leq 360 \mathrm{M}_{\odot}$. We therefore cover the entire predicted mass range of field binaries and the lower end of $\mathrm{IMBH} / \mathrm{IMBH}$ and IMRI binaries. (Our choice of masses is based on the effectualness of the template placement algorithm we use; see Sec. III for details.) Due to the large uncertainty in mass distribution across this range, we will simply assume a uniform rate in $m_{1}$ and $m_{2}$ to calculate net gain. This choice of astrophysical prior weights equal-mass systems as being more likely to occur than asymmetric-mass systems. Since this may bias our results (sub-dominant modes are more significant in higher mass-ratio systems) we will also consider a rate uniform in $M$ and $q$. We will find that BBHs with $M>100 \mathrm{M}_{\odot}$ are the dominant contribution to the net gain using these rate priors, as the sensitive volumes of these larger mass 
systems can be one to two orders of magnitude larger than lower-mass BBHs. Since the existence of IMBHs is more speculative, we additionally report net gains when only stellar-mass BBHs $\left(M<100 \mathrm{M}_{\odot}\right)$ are considered.

The astrophysical rates we use are only meant to be rough approximations for comparisons between template banks; we do not attempt to calculate detection rates. We do, however, report sensitive volumes across the mass space [see Fig. 10. If a particular astrophysical prior is chosen, our results can be used to estimate the total number of non-spinning $\mathrm{BBH}$ detections that will be made in advanced LIGO.

\section{IMPACT OF SUB-DOMINANT MODES ON A DOMINANT MODE TEMPLATE BANK}

Past BBH searches have used waveforms without subdominant modes as templates [6, 7]. Predicted advanced LIGO detection rates are also based on considerations of the dominant mode only [5. This assumes that the effect of neglecting sub-dominant modes is small. To test the validity of this assumption we compare the sensitivity of a template bank without sub-dominant modes to simulated signals without sub-dominant modes and to signals with sub-dominant modes.

We generate one million simulated signals (injections) uniformly distributed in component mass, inclination, sky location, distance, and polarization. Component masses are between 3 and $200 \mathrm{M}_{\odot}$, resulting in total masses $(M)$ between 6 and $400 \mathrm{M}_{\odot}$ and mass ratios $(q)$ between 1 and $\sim 66$. Distance limits are chosen such that an optimally-oriented signal with the same masses as a given injection would produce an SNR between 6 and 100. For each injection we generate a version without sub-dominant modes and a version with sub-dominant modes, both at the same physical distance, using the EOB waveform calibrated to numerical relativity that is described in Ref. 13 ${ }^{11}$ Template waveforms are the dominant-mode version of this waveform.

To calculate the re-weighted SNR of each injection we generate 16 realizations of Gaussian noise colored by the zero-detuned high power noise curve. We use a different set of 16 realizations for each injection. In each realization we calculate the maximum SNR before the injection is added to the noise and after. If the SNR with the injection in the noise is larger than the SNR from noise alone, we record the SNR and calculate $\chi^{2}$ and reweighted SNR. We then find the mean $\rho, \chi^{2}$, and $\tilde{\rho}$ to get a measure of their expectation values. Only realizations that produced an SNR louder than noise are included in the average.

11 Specifically, we use the EOBNRv2 code in the LSC Algorithm Library (LAL) 48 to generate dominant-mode waveforms. For sub-dominant mode waveforms we use the EOBNRv2HM code in LAL.
Due to computational constraints we are not able to calculate re-weighted SNR for every template for every injection. Instead, we use the best matching template from the effectualness study to calculate $\tilde{\rho}$. This is equivalent to maximizing on SNR, then finding re-weighted SNR, which is what current searches do [26]. We note that maximizing on $\tilde{\rho}$ might give better sensitivity, but since this is not what is currently done we do not investigate this idea further.

To calculate the sensitive volume we create 16 bins uniformly distributed in $M$ and 10 bins uniformly distributed in $\log q$, keeping bins which have at least 1000 injections in them. Within each mass bin we create 20 bins uniform in $\log$ distance. We find the efficiency in each distance bin, then numerically integrate over the distance bins to get the sensitive volume. The distance bins span the smallest injected distance to the largest within the given mass bin. For distances smaller then the closest injection we assume $\epsilon=1$; for distances larger than the furthest injection we assume $\epsilon=0$.

\section{A. Effectualness of the dominant-mode bank}

Past BBH searches [6, 7, have constructed template banks by placing templates in parameter space such that no more than $3 \%$ of SNR is lost due to the discreetness of the bank. In other words, templates are placed such that the minimal match, which is the minimum of the effectualness of the bank, is $\geq 97 \%$. Templates are placed in a hexagonal grid [49] using a metric calculated from the mismatch between a waveform with intrinsic parameters $\boldsymbol{\Upsilon}$ and a waveform with parameters $\boldsymbol{\Upsilon}+\delta \boldsymbol{\Upsilon}$ [11, 12. This metric is found analytically using inspiral waveforms expanded to 1.5 post-Newtonian order (PN) in phase [50] ${ }^{12}$ As this metric is based solely on the inspiral part of the waveform, it will fail to properly place templates at high mass, where the waveforms are dominated by merger and ringdown [51. More sophisticated template placement methods have recently been devised: a new metric has been calculated using the $3.5 \mathrm{PN}$ approximant [52] and a "stochastic" placement method has been developed that uses no metric [53. In this study we are only interested in the relative difference in sensitivity when a template bank neglects sub-dominant modes and not on the most efficient placement algorithm. We therefore use the same 1.5 PN placement algorithm that has been used in prior searches ${ }^{13}$ putting a cut on total

12 In Ref. [50, the metric was calculated to second post-Newtonian order. However, a bug was recently discovered in the templateplacement code in LAL (on which this study depends) that caused the metric calculation to be truncated at 1.5 PN. Since this bug was discovered after we had finished all calculations, we simply use the $1.5 \mathrm{PN}$ metric.

13 We emphasize that while the templates are placed using the 1.5 PN approximant, the template waveforms are EOBNRv2. 


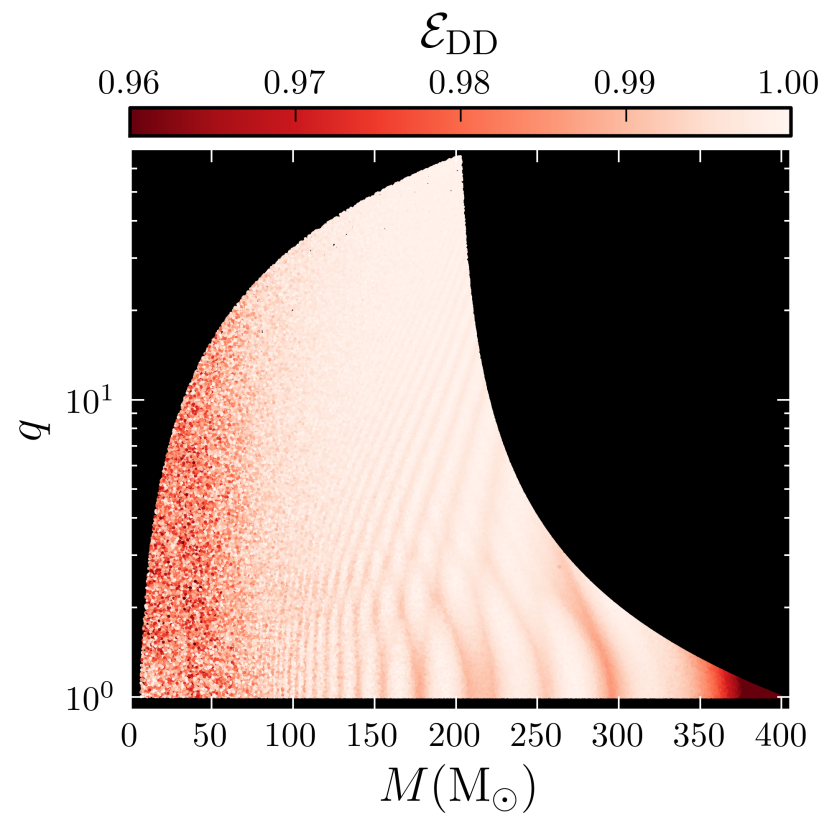

FIG. 1. Effectualness of a template bank placed using the $1.5 \mathrm{PN}$ metric and using waveforms without subdominant modes (EOBNRv2) to injections without subdominant modes (also EOBNRv2), $\mathcal{E}_{\mathrm{DD}}$. The desired minimal match of the bank $(=\min \{\mathcal{E}\})$ is 0.97 .

mass where the metric fails to maintain the desired $97 \%$ minimal match.

To determine where the $1.5 \mathrm{PN}$ metric fails to maintain a $97 \%$ minimal match we generate a template bank to cover component masses between 3 and $200 \mathrm{M}_{\odot}(6 \leq$ $\left.M / \mathrm{M}_{\odot} \leq 400\right)$. Using the aLIGO zero-detuned high power PSD, this results in a bank of 19800 templates. We calculate the effectualness of this bank to the injections without sub-dominant modes, $\mathcal{E}_{\mathrm{DD}}$; the results are shown in Fig. 1. The effectualness appears to be $>0.97$ for $M \lesssim 360 \mathrm{M}_{\odot}$, but is lower than that for larger $M$. Indeed, we find that less than $0.3 \%$ of injections with $M<360 \mathrm{M}_{\odot}$ have an effectualness $<0.97$ [see Fig. 3, but $\sim 70 \%$ of injections have an effectualness $<0.97$ for total masses larger than $360 \mathrm{M}_{\odot}$. We therefore place a cutoff of $M<360 \mathrm{M}_{\odot}$ when reporting effectualness and efficiency.

\section{B. Efficiency to injections with sub-dominant modes}

Figures 2 and 3 show the effectualness of the dominantmode bank when sub-dominant modes are added to the injections, $\mathcal{E}_{\mathrm{DS}}$. We find that $\mathcal{E}_{\mathrm{DS}}$ is substantially lower than $\mathcal{E}_{\mathrm{DD}}$ for many injections: $\sim 50 \%$ of sub-dominant injections have an effectualness $<0.97$ for $M<360 \mathrm{M}_{\odot}$. In Fig. 2 we see that the effectualness decreases with increasing mass ratio. This is expected as the amount

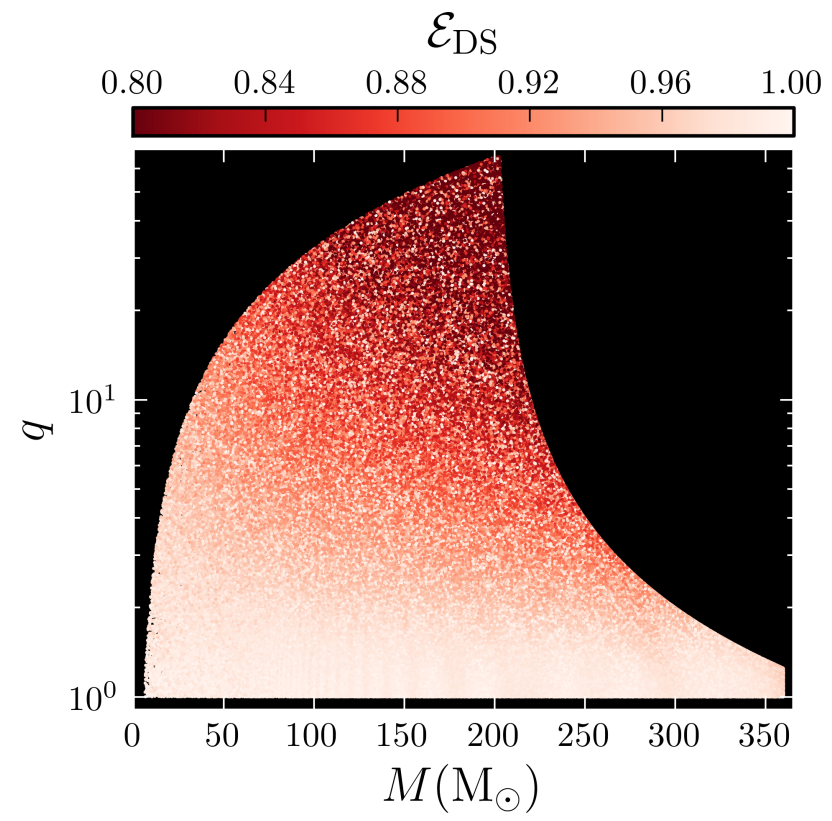

FIG. 2. Effectualness of the $1.5 \mathrm{PN}$ dominant-mode template bank to injections with sub-dominant modes, $\mathcal{E}_{\mathrm{DS}}$. The desired minimal match $(=\min \{\mathcal{E}\})$ of the bank was 0.97 , but we see that the effectualness drops below this as we go to higher mass ratio.

of power in the higher modes will increase relative to the dominant mode as the binary becomes more asymmetric.

Given that $\mathcal{E}_{\mathrm{DS}}<\mathcal{E}_{\mathrm{DD}}$ we wish to know if the predicted detection rates for advanced LIGO will be worse than expected. As discussed in Sec. IIC we cannot tell this from effectualness alone. Instead, we compute $\mathcal{G}_{\mathrm{DD}}^{\mathrm{DS}}$, which is the sensitive volume of injections with sub-dominant modes $\left(V_{\mathrm{DS}}\right)$ relative to the sensitive volume of injections without sub-dominant modes $\left(V_{\mathrm{DD}}\right)$ when both are recovered by the dominant-mode template bank. If $\mathcal{G}_{\mathrm{DD}}^{\mathrm{DS}}<1$ it means that the sensitivity to real signals (which have sub-dominant modes) will be worse than predicted. If $\mathcal{G}$ DD $>1$ it means that the sensitivity to real signals will be better than predicted; if $\mathcal{G}$ DD $=1$ it means there is no difference.

Figure 4 shows $\mathcal{G}_{\mathrm{DD}}^{\mathrm{DS}}$ in each mass bin. We see that the gain $\approx 1$ everywhere. Indeed, if we assume a uniform astrophysical rate in component masses we find net $\left\{\mathcal{G}_{\mathrm{DD}}^{\mathrm{DS}}\right\}=1.005 \pm 0.004$; a uniform rate in $M$ and $q$ yields net $\left\{\mathcal{G}_{\mathrm{DD}}^{\mathrm{DS}}\right\}=1.019 \pm 0.004$. This means that a dominant-mode template bank yields predicted detection rates even though both the bank and the predictions neglect sub-dominant modes.

Comparing Fig. 4 to Fig. 2 we see that $\mathcal{G}_{\mathrm{DD}}^{\mathrm{DS}} \approx 1$ despite the drop in effectualness to injections with subdominant modes. Notably, the relative gain is slightly larger than 1 in the areas where the effectualness is smallest. This means that the drop in effectualness seen in Fig. 2 is largely due to an increase in the amount of power in 


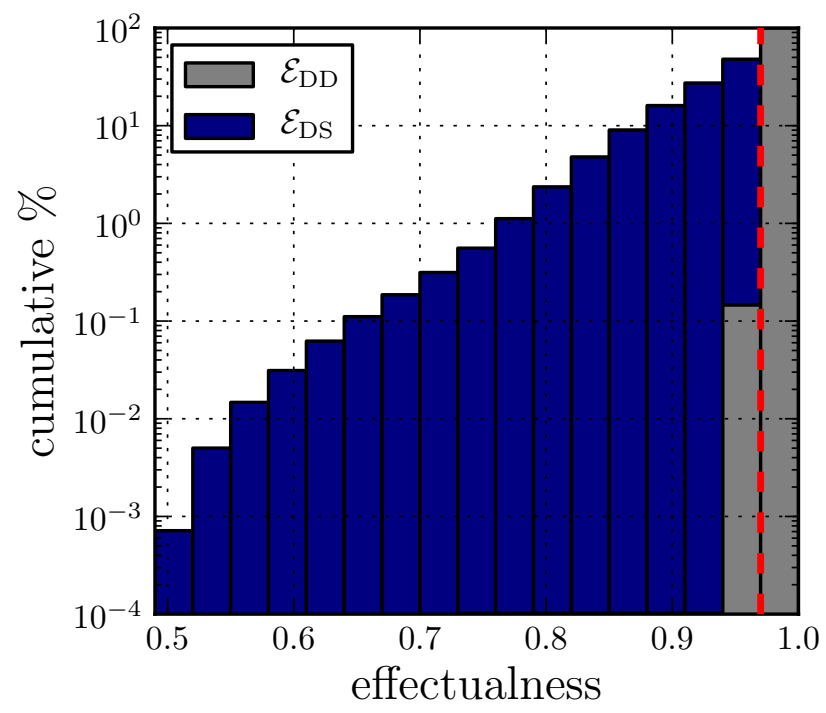

FIG. 3. Cumulative histogram of the effectualness of a template bank without sub-dominant modes to injections without sub-dominant modes $\mathcal{E}_{\mathrm{DD}}$ (gray) and injections with subdominant modes $\mathcal{E}_{\mathrm{DS}}$ (blue). Each bin gives the fraction of injections with an effectualness less than the right edge of the bin. The red dashed line indicates the target minimal match for the bank (0.97). All injections have total mass $<360 \mathrm{M}_{\odot}$.

injections when sub-dominant modes are added rather than a decrease in SNR between the templates and injections.

A decrease in effectualness will always result in an increase in $\chi^{2}$, regardless of whether the mismatch $(\equiv 1-\mathcal{E})$ results in an increase or decrease in SNR. Whether reweighted SNR increases or decreases therefore depends on whether the gain in $\rho$ offsets the gain in $\chi^{2}$. Figure 5 shows reduced $\chi^{2}$ versus SNR for all injections with $M<360 \mathrm{M}_{\odot}$. The black arrows indicate how, on average, the injections move in this plane when higher modes are added. The solid black line shows where $\tilde{\rho}=8$, which is the threshold we use when calculating efficiency. All injections to the left of this line are missed, all injections to the right are found. We see that at this threshold the increase in $\chi^{2}$ is roughly offset by the increase in SNR when sub-dominant modes are added, which explains why $\mathcal{G}_{\mathrm{DD}}^{\mathrm{DS}} \approx 1$ despite an increase in mismatch for injections with sub-dominant modes.

Figure 5 also shows that as we go to higher $\rho$, the gain in $\chi_{r}^{2}$ becomes larger relative to the gain in SNR when sub-dominant modes are included (the black arrows progressively point to higher $\chi_{r}^{2}$ rather than to higher SNR as we move to the right in the plot). If our threshold $\tilde{\rho}$ were larger more injections would move below threshold (from an increase in $\chi^{2}$ ) than above (from an increase in $\rho$ ). This is confirmed by Fig. 6. The top plot shows the percentage of injections that move above threshold (gained) and the percentage of injections that move below threshold (lost) when sub-dominant modes are added, as a function of threshold $\tilde{\rho}$. Around a threshold of $\tilde{\rho}=8$ there is a small net gain in the number of injections found when sub-dominant modes are added. If the threshold were larger than $\sim 9.5$, however, there would be a net loss. This loss causes an overall drop in efficiency, which in turn causes a drop in sensitivity. For example, the bottom plot in Fig. 6 shows $\mathcal{G}_{\mathrm{DD}}^{\mathrm{DS}}$ when the threshold $\tilde{\rho}=11$. We see that many tiles have gains $<1$ now. Assuming a uniform astrophysical rate in component-masses yields net $\left\{\mathcal{G}_{\mathrm{DD}}^{\mathrm{DS}}\right\}=0.994 \pm 0.004$; uniform in $M, q$ yields net $\left\{\mathcal{G}_{\mathrm{DD}}^{\mathrm{DS}}\right\}=0.988 \pm 0.006$. Note, however, that the decrease in gain mostly occurs for tiles with $M>100 \mathrm{M}_{\odot}$ and $q>4$, which is where the drop in effectualness is the largest. If we restrict our net gain calculation to stellar-mass BBHs only $\left(M<100 \mathrm{M}_{\odot}\right)$ we get net $\left\{\mathcal{G}_{\mathrm{DD}}^{\mathrm{DS}}\right\}=1.01 \pm 0.03$ for both rate priors.

We conclude that if gravitational waves can be detected at a single-detector $\tilde{\rho} \approx 9.5$ or less, then neglecting sub-dominant modes will not affect the expected sensitivity of a non-spinning BBH search across all masses. If the threshold for detection is larger, then neglecting subdominant modes will cause a larger drop in sensitivity than what would otherwise be expected for BBHs with $q \gtrsim 4$ and containing at least one $\mathrm{IMBH}\left(m \gtrsim 100 \mathrm{M}_{\odot}\right)$. Non-spinning stellar-mass BBHs, however, are not affected by neglecting sub-dominant modes, even if the single-detector threshold for detection is $\tilde{\rho}=11$.

\section{PREDICTED EFFICIENCY OF A TEMPLATE BANK WITH SUB-DOMINANT MODES}

In the previous section we found that neglecting subdominant modes did not adversely affect the sensitivity of a search for non-spinning BBHs. We found this by comparing the sensitive volume of injections with subdominant modes to the sensitive volume of injections without sub-dominant modes when both are recovered by dominant-mode templates. However, the decrease in effectualness to injections with sub-dominant modes means that there is some power not being recovered by the dominant-mode templates. If the templates had sub-dominant modes they would be able to recover that power, which could increase the sensitivity of the search. We therefore wish to know how the sensitivity of a bank of templates with sub-dominant modes compares to a bank of templates without sub-dominant modes, $\mathcal{G}_{\mathrm{DS}}^{\mathrm{SS}}$.

\section{A. Search sensitivity when sub-dominant modes are included in all templates}

Currently, no search exists that uses a bank of templates with sub-dominant modes. We do not try to create one here. Instead, we simulate how such a search would perform as compared to the dominant-mode template bank used here. In our simulation we will continue 


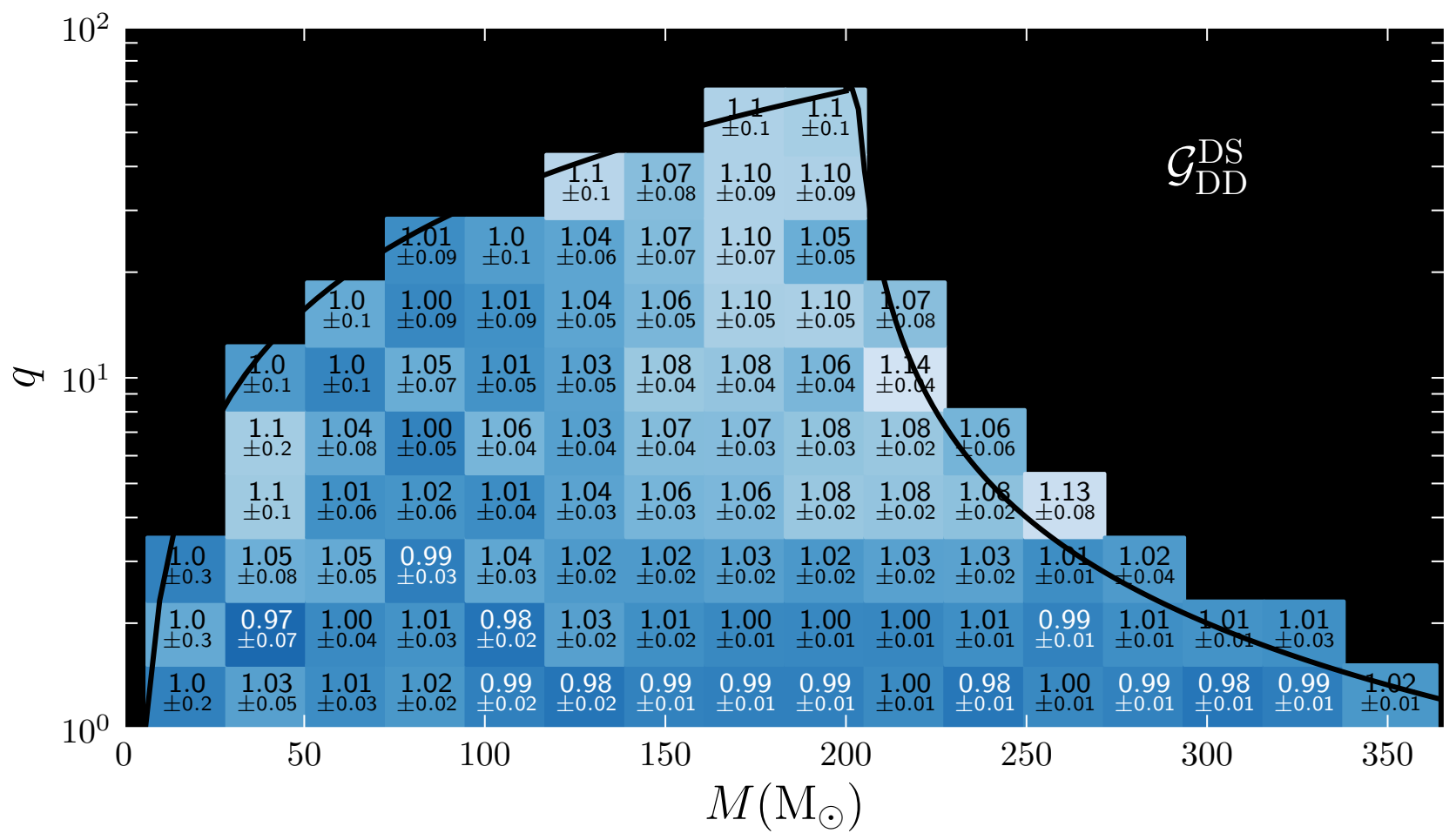

FIG. 4. Sensitive volume of injections with sub-dominant modes relative to sensitive volume of injections without sub-dominant modes when both are recovered by the dominant-mode template bank ( $\mathcal{G}_{\mathrm{DD}}^{\mathrm{DS}}$; see Sec. IIC for definition) in $M, q$ plane. The solid black line indicates the injection region. Only tiles with 1000 or more injections in them are shown.

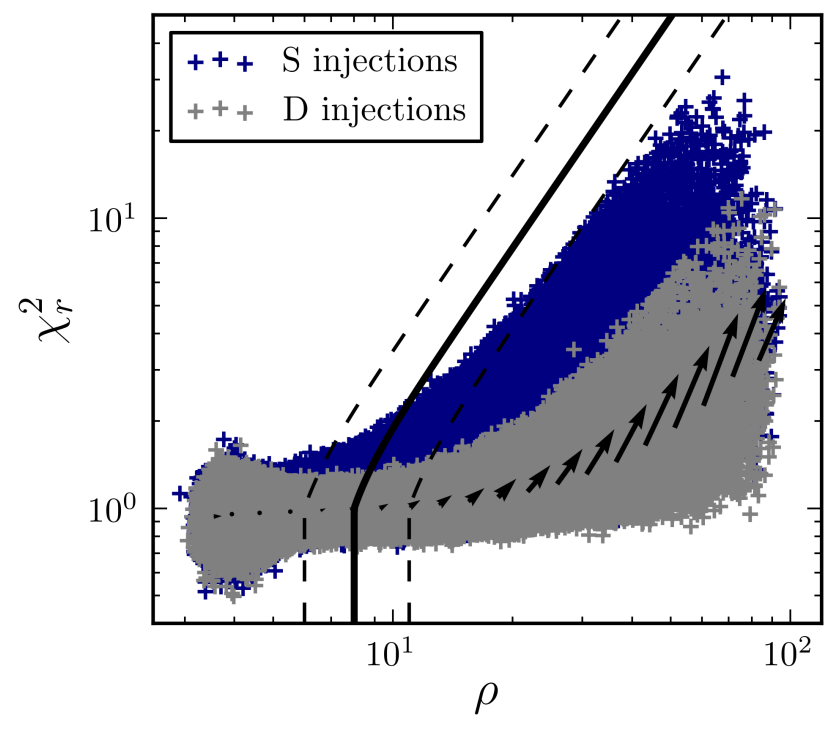

FIG. 5. Reduced $\chi^{2}\left(\chi_{r}^{2}\right)$ versus SNR of all injections with $M<360 \mathrm{M}_{\odot}$. Gray crosses are injections with the dominantmode only, dark-blue crosses are injections with sub-dominant modes. The dashed and sold black lines show lines of constant $\tilde{\rho}$; shown are $\tilde{\rho}=6,8$, and 11 (dashed, solid, and dashed, respectively). The black arrows indicate where, on average, injections move in this plane when sub-dominant modes are added. to use $\tilde{\rho}$ maximized over the template bank as our detection statistic. We do not try to find the optimal search statistic when sub-dominant modes are used. As discussed in Sec. II C 1, finding the optimal statistic over a bank of templates is difficult, if not impossible, due to our lack of knowledge of the mass distribution of BBHs. Our goal here is to answer the question: using current search techniques, will sensitivity improve if we include sub-dominant modes in templates?

To calculate $\mathcal{G}_{\mathrm{DS}}^{\mathrm{SS}}$ we need to know the sensitive volume of a template bank with sub-dominant modes to injections with sub-dominant modes, $V_{\mathrm{SS}}$. Since $V_{\mathrm{SS}}$ depends on the number of injections found by the template bank above some threshold, finding $V_{\text {SS }}$ requires two pieces of information: the average re-weighted SNR of injections filtered with a sub-dominant mode template bank $\left\langle\tilde{\rho}_{\mathrm{SS}}\right\rangle$, and the threshold for making a detection in such a search.

In order to estimate $\left\langle\tilde{\rho}_{\mathrm{SS}}\right\rangle$ of each injection we first calculate the maximum SNR that can be recovered from the injection by finding the overlap of the injection with itself [see Eq. [14)]. A real sub-dominant mode search will use discrete templates, as is done with the dominantmode bank. The discreteness of the template bank will cause some loss of SNR. Even though both the injections and templates have sub-dominant modes in our simulation, the mismatch due to the discreteness of the bank will also cause some small increase in $\chi_{r}^{2}$, as was the 

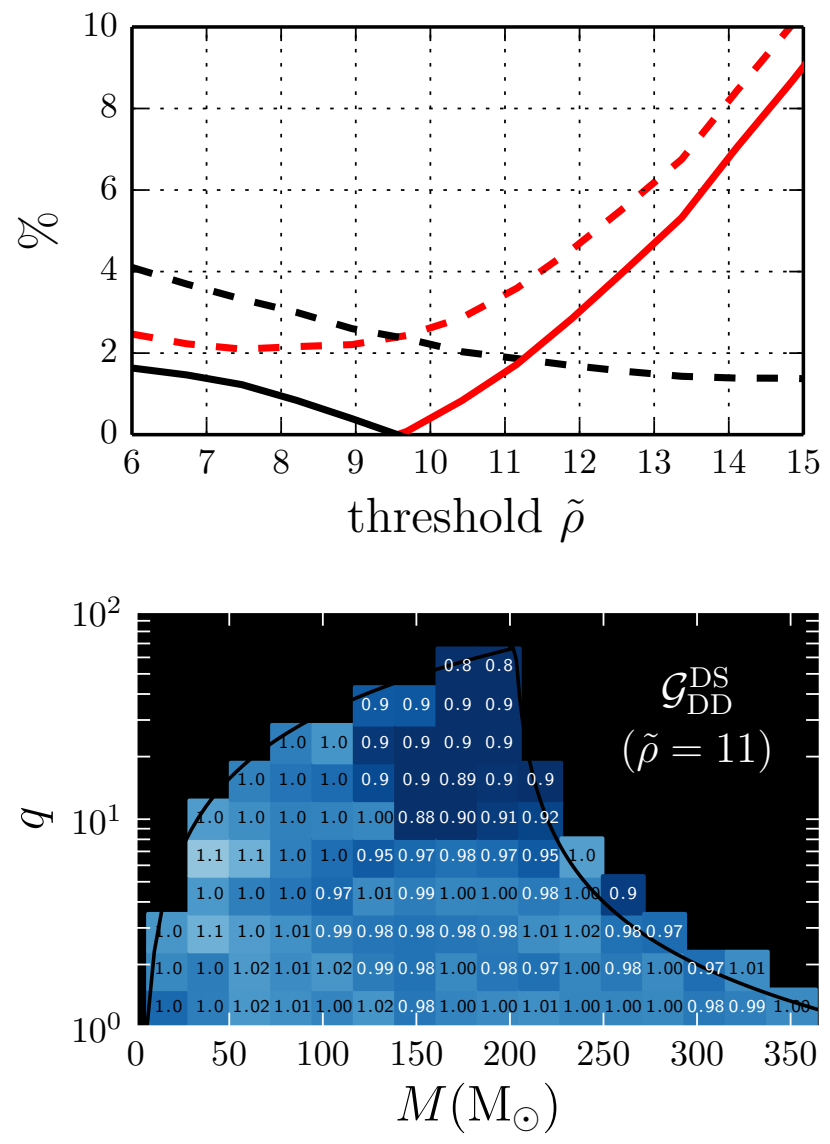

FIG. 6. Top: Percentage of injections gained and lost when sub-dominant modes are added as a function of threshold $\tilde{\rho}$. The dashed black line shows the percentage of injections gained, the dashed red line shows the percentage of injections lost. The solid line shows the net gain/loss; where it is black there is a net gain; where it is red there is a net loss. Bottom: $\mathcal{G}_{\text {DD }}^{\text {DS }}$ when the threshold re-weighted SNR for both D and S injections is 11 instead of 8 . The solid black line indicates the injection region. Errors on gain are approximately twice the errors reported in the corresponding tiles in Fig. 4

case for the dominant-mode injections recovered by the dominant-mode template bank (gray crosses in Fig. 5). This in turn causes a small decrease in re-weighted SNR relative to SNR for some injections. To simulate both of these effects, we estimate $\left\langle\tilde{\rho}_{\mathrm{SS}}\right\rangle$ to be:

$$
\left\langle\tilde{\rho}_{\mathrm{SS}}\right\rangle=\frac{\left\langle\tilde{\rho}_{\mathrm{DD}}\right\rangle}{\left\langle\rho_{\mathrm{DD}}\right\rangle} \mathcal{E}_{\mathrm{DD}} \max \left\{\rho_{\mathrm{SS}}\right\} .
$$

Here $\mathcal{E}_{\mathrm{DD}}$ simulates the loss in SNR due to the discreteness of the bank - i.e., we have assumed $\left\langle\rho_{\mathrm{SS}}\right\rangle \approx$ $\mathcal{E}_{\mathrm{DD}} \max \left\{\rho_{\mathrm{SS}}\right\}$ - and $\left\langle\tilde{\rho}_{\mathrm{DD}}\right\rangle /\left\langle\rho_{\mathrm{DD}}\right\rangle$ simulates the effect of $\chi_{r}^{2}$ re-weighting. Thus the discreteness of our simulated template bank with sub-dominant modes is equivalent to the discreteness of our (real) dominant-mode bank.

By assuming $\left\langle\rho_{\mathrm{SS}}\right\rangle \approx \mathcal{E}_{\mathrm{DD}} \max \left\{\rho_{\mathrm{SS}}\right\}=\mathcal{E}_{\mathrm{DD}} \sqrt{\left\langle h_{\mathrm{S}}, h_{\mathrm{S}}\right\rangle}$ in Eq. 22 we have neglected any contribution to the
SNR from noise. Due to the maximization over the phase, the average SNR of a simulated signal $h$ when filtered with itself in Gaussian noise will be slightly larger than $\sqrt{\langle h, h\rangle}$. Rather than adding a factor to our estimate of $\left\langle\tilde{\rho}_{\mathrm{SS}}\right\rangle$ to account for this contribution, when finding $\mathcal{G}_{\mathrm{DS}}^{\mathrm{SS}}$ we use a "noiseless" estimate of $\left\langle\tilde{\rho}_{\mathrm{DS}}\right\rangle$, given by:

$$
\left\langle\tilde{\rho}_{\mathrm{DS}}\right\rangle^{\text {(noiseless) }}=\frac{\left\langle\tilde{\rho}_{\mathrm{DS}}\right\rangle}{\left\langle\rho_{\mathrm{DS}}\right\rangle} \mathcal{E}_{\mathrm{DS}} \max \left\{\rho_{\mathrm{SS}}\right\} .
$$

Here, $\mathcal{E}_{\mathrm{DS}} \max \left\{\rho_{\mathrm{SS}}\right\}$ gives $\left\langle\rho_{\mathrm{DS}}\right\rangle$ with the noise contribution removed and $\left\langle\tilde{\rho}_{\mathrm{DS}}\right\rangle /\left\langle\rho_{\mathrm{DS}}\right\rangle$ accounts for $\chi_{r}^{2}$ reweighting.

When calculating the sensitivity of the dominant-mode template bank we used a threshold of $\tilde{\rho}=8$. In a real search triggers are produced by gravitational waves and by background noise. High statistical significance is therefore required in order for a trigger to be considered a gravitational-wave candidate. The standard measurement of significance is false-alarm probability $\mathcal{F}(\rho)$. When evaluating the sensitivity of the dominant-mode template bank we choose $\tilde{\rho}=8$ because it corresponds to a false-alarm probability small enough that we could confidently claim detection in real detector data. This means that to compare the sensitivity of the simulated sub-dominant mode search to the dominant-mode search we must chose a threshold $\tilde{\rho}$ that results in the same falsealarm probability that $\tilde{\rho}=8$ did in the dominant-mode search.

As discussed in Sec. II sub-dominant modes break the degeneracy between $\theta, \phi$ and $\kappa$. This means that to fully recover all of the power in the sub-dominant modes we have to maximize over three extrinsic parameters instead of one. These additional maximizations will cause the single-detector SNR distribution in noise to change. SNR from dominant-mode templates is $\chi$ distributed with 2 degrees of freedom in stationary Gaussian noise. If a template has sub-dominant modes, the SNR will have a larger number of degrees of freedom. The increase in the degrees of freedom means that $\mathcal{F}(\rho)$ at a given SNR will increase. In order to keep the same falsealarm probability for a bank with sub-dominant modes as we had for the dominant-mode bank, the threshold $\tilde{\rho}$ must therefore increase. The relative gain in sensitivity of a template bank with sub-dominant modes depends on whether the increase in SNR due to the sub-dominant modes is enough to offset the increase in SNR threshold.

In Appendix $\mathrm{B}$ we estimate the false-alarm probability of a dominant-mode template bank with $N$ templates $\mathcal{F}(\rho \mid \mathrm{D}, N)$ at $\rho=8$ [see Eq. (B2)]. For the dominant-mode bank used here $N=19800$, which gives $\mathcal{F}(\rho=8 \mid \mathrm{D}, N) \approx 2.5 \times 10^{-10}$. We also calculate falsealarm probability as a function of SNR of the simulated sub-dominant mode bank $\mathcal{F}(\rho \mid \mathrm{S})$ [see Eqs. (B1] and (B6)]. Assuming $\tilde{\rho} \approx \rho$ for injections, we estimate that the threshold $\tilde{\rho}$ would have to increase to 8.31 for the sub-dominant mode bank in order for $\mathcal{F}(\rho \mid \mathrm{S}, N)$ to also be equal to $2.5 \times 10^{-10}$. 
Using the estimated $\tilde{\rho}_{\text {SS }}$ given in Eq. 22 and a threshold $\tilde{\rho}=8.31$ we calculate the sensitive volume of the simulated sub-dominant mode template bank $V_{\mathrm{SS}}$ in the same $M, q$ tiles we used in Fig. 4. We compare this to $V_{\mathrm{DS}}$ (which used a threshold $\tilde{\rho}=8$ ) to get $\mathcal{G}_{\mathrm{DS}}^{\mathrm{SS}}$; this is plotted in Fig. 7. We find that the sub-dominant mode template bank has worse sensitivity then the dominantmode bank across nearly the entire mass-space, dropping to as low as $\sim 90 \%$ for mass ratios between 1 and 1.5 .

The reason for the poor sensitivity of the sub-dominant mode bank to equal-mass systems can be understood by considering Fig. 2, in which we see that when $q \rightarrow 1$, $\mathcal{E}_{\mathrm{DS}} \rightarrow 1$. This means that dominant-mode templates are recovering nearly all of the available power in equalmass signals. Put another way, signals from equal-mass systems gain almost no SNR by adding sub-dominant modes to the templates. The SNR distribution in noise of sub-dominant mode templates that are close to the equalmass line are not much different than their dominantmode counterparts. However, false-alarm probability is a global property of the template bank: even though the SNR distribution does not change for equal-mass templates, it does for more asymmetric-mass templates. The presence of these templates in the bank affects the falsealarm probability of the entire search. The result is that equal-mass signals suffer an increase in threshold but gain no SNR from the sub-dominant template bank.

The sub-dominant mode bank does have better sensitivity at higher mass ratios and total masses. In the highest mass-ratio tile in Fig. 2 the sensitivity is twice that of a dominant-mode bank. A sub-dominant mode bank may therefore still increase the probability of making a detection, if the total gain in sensitive volume to higher mass-ratio systems is enough to offset the drop in sensitivity to equal-mass systems. However, assuming either a uniform astrophysical rate in $m_{1}, m_{2}$ or a uniform rate in $M, q$ yield net gains $<1$ : the former gives net $\left\{\mathcal{G}_{\mathrm{DS}}^{\mathrm{SS}}\right\}=0.932 \pm 0.003$ while the latter gives net $\left\{\mathcal{G}_{\mathrm{DS}}^{\mathrm{SS}}\right\}=0.987 \pm 0.003$. Restricting to stellar-mass $\mathrm{BBHs}$ also results in net gains $<1$, yielding $0.92 \pm 0.01$ and $0.96 \pm 0.01$ for the two priors, respectively.

Using templates that more accurately model signals should improve sensitivity. The reason the sub-dominant mode templates do not is due to the astrophysical prior that is inherent in the search. Simply selecting the template with the largest SNR when maximizing over the bank (as we have done for both the dominant-mode and sub-dominant mode bank, and as is done in current searches) assumes that every template is equally likely to detect a signal. The detectors are not equally sensitive to all signals, however, nor is the density of templates uniform. This makes the search most sensitive to a particular astrophysical rate distribution. Adding subdominant modes changes the distribution to which the search is most sensitive, thereby implicitly changing the search prior.

As noted by Refs. 14 and 15, the largest SNR increase occurs for signals from asymmetric-mass binaries and from systems inclined to the line of sight. Yet the magnitude of the detector's sensitivity is lowest to these systems. Including sub-dominant modes while weighting the SNR of each template equally causes us to gain sensitivity to signals for which we are least sensitive at the cost of losing sensitivity to signals for which we are most sensitive. We can see this negative correlation in Fig. 8, which shows the fractional gain in SNR when sub-dominant modes are added (ignoring the effects of $\chi_{r}^{2}$ re-weighting) versus the distance at which a signal can be detected by the dominant-mode bank at SNR 8 ("sensitive distance"), colored by mass-ratio. The sensitive distance of the signals with the largest gain (and mass-ratio) is an order of magnitude smaller than the sensitive distance of the signals with the smallest gain. In general, a plot such as this can be used to determine whether changing the parameters of a search are worthwhile. If there is a negative correlation between SNR gain and sensitive distance - i.e., only signals for which the detector is least sensitive gain SNR by changing the search parameters - that change is unlikely to improve the overall detection rate unless the astrophysical distribution is strongly weighted to those signals.

\section{B. Splitting the template bank to improve sensitivity}

Including sub-dominant modes in templates would improve the sensitivity of the search if we applied a weight to each template that better reflected the probability the template will make a detection. Determining the best weight to use requires knowledge of the astrophysical rate of signals. We expect that signals will be distributed uniformly in the inclination angle $\theta$. However, the proper weight to apply to signals with this assumption is already inherent in the SNR. The projection of the waveform into the radiation frame, and then again into the detector's frame (done by the $\mathbf{P}$ and $\mathbf{K}$ matrices, respectively, in Appendix A is the weight one should apply to the SNR assuming signals are distributed uniformly in space. Indeed, that the SNR from an inclined signal is smaller than the SNR from a face-on signal at the same distance is a result of the antenna pattern indicating that these signals are less likely to be detected.

Any weight we apply to the SNR must therefore be based on the distribution of intrinsic paramters. Given the large uncertainty in the mass distribution of $\mathrm{BBHs}$ this is difficult to do. However, if we can find a weight that results in $\mathcal{G} \geq 1$ relative to the dominant-mode search in all mass tiles, we know that this new search will be at least as sensitive to real signals as the dominantmode search, regardless of the astrophysical mass distribution.

The simplest way to arrive at such a weight is to split the template bank in two parts. In one part we use templates with sub-dominant modes; in the other, we use dominant-mode templates. In this scenario false-alarm 


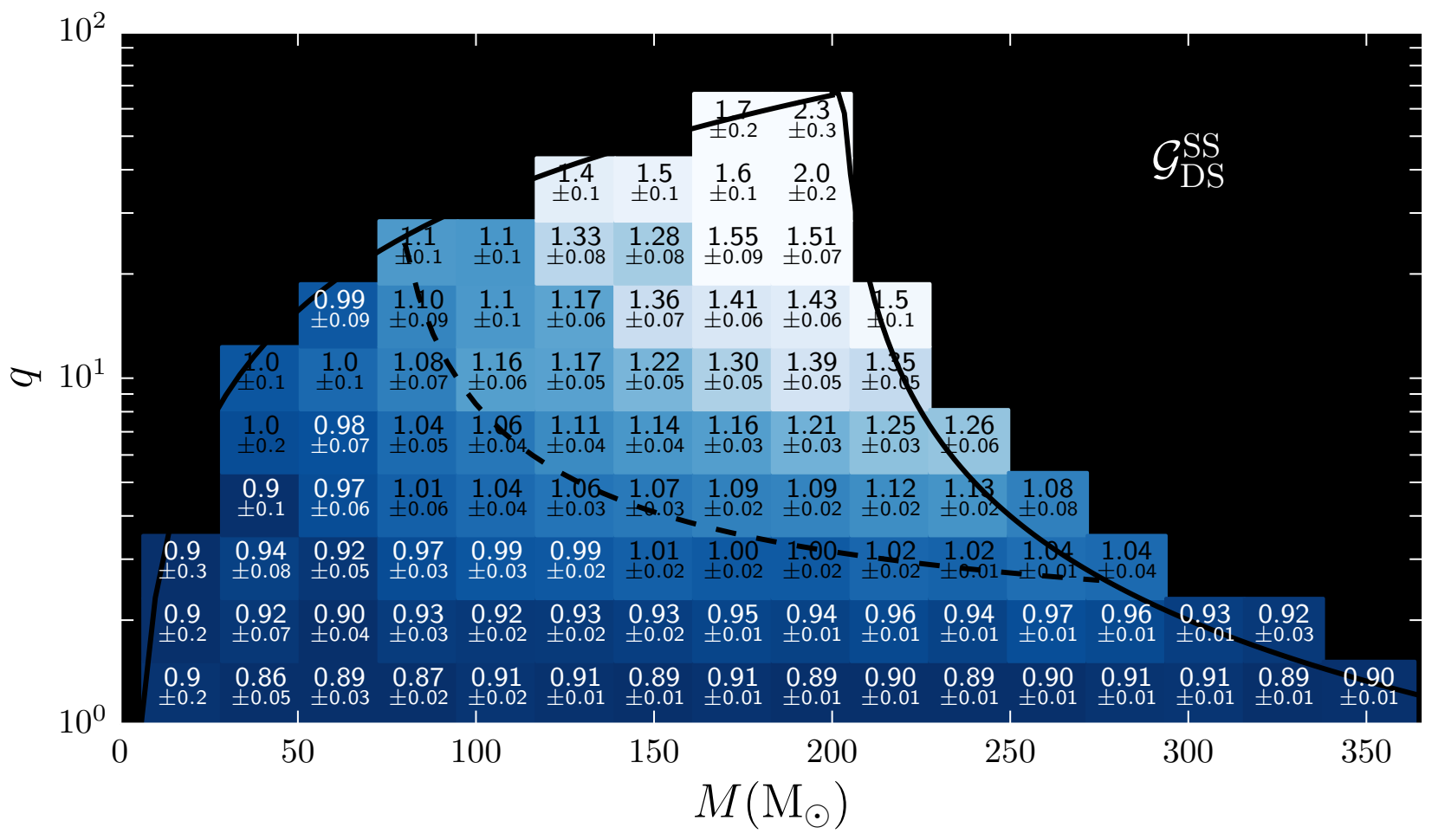

FIG. 7. Relative gain of sub-dominant bank compared to dominant-mode bank if the sub-dominant modes are used everywhere, $\mathcal{G}_{\mathrm{DS}}^{\mathrm{SS}}$. The solid black line indicates the injection region. The dashed black line shows where we choose to place a boundary between dominant-mode and sub-dominant mode templates for a split-bank search (see Fig. 9 for gain).

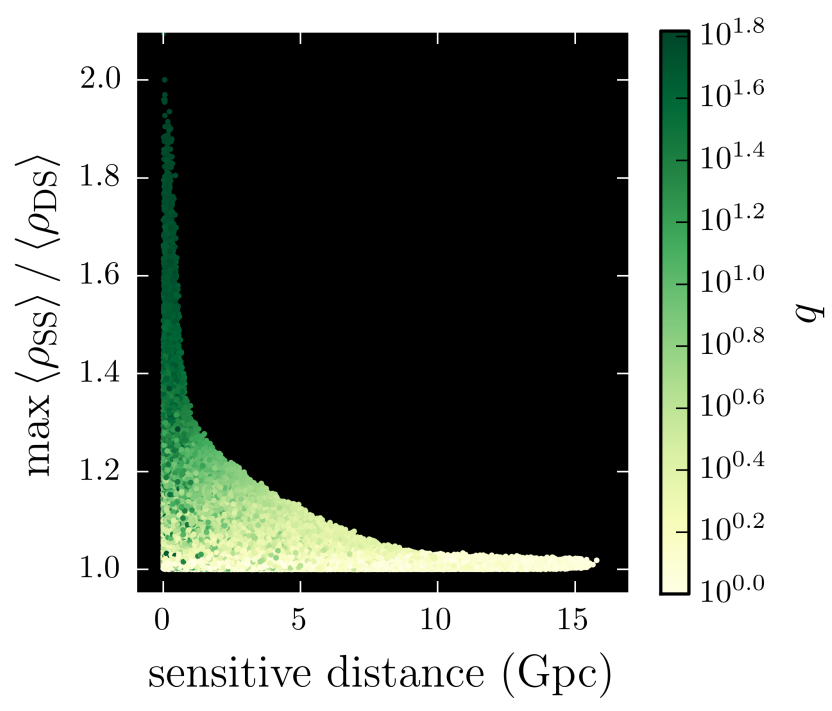

FIG. 8. The maximum gain in SNR that is possible by adding sub-dominant modes to templates $\left(\max \left\langle\rho_{\mathrm{SS}}\right\rangle /\left\langle\rho_{\mathrm{DS}}\right\rangle\right)$ versus the distance at which a signal can be detected by the dominant-mode template bank at SNR 8 (sensitive distance). Each point represents a simulated signal; the points are colored by their mass-ratio $(q)$. probabilities are calculated separately in each region, then combined. As we will see below, the process of combining the results across the split effectively downweights higher mass-ratio templates such that they do not hurt the sensitivity to equal-mass systems, yet still improves sensitivity to the high-mass ratio signals.

In order to not lose any sensitivity we need to place the split between dominant-mode and sub-dominant mode templates at points in the mass space where $\mathcal{G}_{\mathrm{DS}}^{\mathrm{SS}}>1$. Otherwise, signals with masses that are in the subdominant part of the bank that are near the split will suffer a loss of sensitivity. We empirically chose to split the bank such that sub-dominant mode templates are used when 14

$$
m_{2}<0.6 m_{1}-43 \text {. }
$$

This cut is shown in Fig. 7 (black dashed line). We note that this is an empirical estimate for this template bank and Gaussian noise. If a real search were performed, this cut would have to be tuned based on the noise and bank characteristics. We assume that such a cut would be in approximately the same location in parameter space as the cut we choose here, however.

\footnotetext{
${ }^{14}$ We put the cut in $m_{1}, m_{2}$ rather then $M, q$ because we found that the $\mathcal{G}_{\mathrm{DS}}^{\mathrm{SS}}$ was roughly linear in $m_{1}, m_{2}$.
} 
Using the split in Eq. 24 we find that 3750 templates are in the sub-dominant region while 16050 templates remain in the dominant-mode region. In Appendix B we find that $\mathcal{F}(\rho)$ is roughly proportional to the number of templates in the search [see Eq. (B1)]. This means that the false-alarm probabilities we get for triggers in the dominant-mode part of the template bank will have decreased by a factor of $16050 / 19800 \approx 0.8$ compared to what we get when we search the entire mass space. Likewise, the false-alarm probability of triggers in the sub-dominant mode part of the bank will decrease by a factor of $\sim 0.2$. These drops in false-alarm probabilities are artificial: they are due solely to our choice of cut. Indeed, we could split the bank an arbitrary number of times, thereby arbitrarily decreasing false-alarm probabilities.

To account for this artificial decrease we must combine results by multiplying the signals' false alarm probabilities by a trials factor [6]. For the dominant-mode part of the bank we multiply by $19800 / 16050 \approx 1.2$; this is equivalent to using dominant mode templates throughout the entire mass space, and so the threshold in this part of the bank remains $\tilde{\rho}=8$. In the sub-dominant mode part of the bank we multiply by $19800 / 3750 \approx 5.3$. To keep $\mathcal{F}(\rho)$ fixed at $2.5 \times 10^{-10}$, the threshold in this region increases to 8.44. This is equivalent to down-weighting $\tilde{\rho}$ of templates in this part of the bank, as desired.

Figure 9 shows the relative gain of the split bank (indicated by $\frac{\mathrm{S}}{\mathrm{D}}$ ) compared to using dominant mode templates everywhere $\left(\mathcal{G}_{\mathrm{DS}}^{\frac{\mathrm{S}}{\mathrm{D}} \mathrm{S}}\right)$. We find that we can gain sensitivity by using templates with sub-dominant modes if we split the bank using the criteria in Eq. (24). Since dominant-mode templates are used below this cut, we lose no sensitivity relative to what we had when we used dominant-mode templates everywhere. In terms of possible astrophysical systems, note that only IMRIs $\left(m_{1} \gtrsim 100 \mathrm{M}_{\odot}, m_{2} \lesssim 80 \mathrm{M}_{\odot}\right)$ fall in the region where sub-dominant modes are used, and that the dominantmode part of the bank covers all possible stellar-mass BBHs $\left(m_{1}, m_{2} \lesssim 80 \mathrm{M}_{\odot}\right)$.

Splitting the bank and only using sub-dominant modes in one part results in a search that is as good or better than the dominant-mode search in all mass tiles, as we desired. The net gain, however, is not much larger than simply using dominant-mode templates everywhere. Assuming a uniform astrophysical rate in component masses

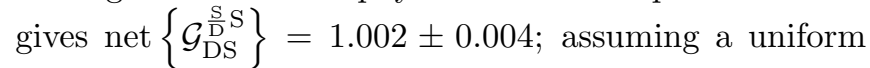
rate in $M, q$ yields $1.024 \pm 0.004$. The reason for the nearly non-existent increase in net gains can be understood by considering Fig. 10, which shows the sensitive volume of the split bank in $\mathrm{Gpc}^{3}$. For a given total mass, the largest sensitivity occurs at equal mass by a large margin. For example, between $\sim 190$ and $200 \mathrm{M}_{\odot}$ (the mass bin with the largest range in $q$ ) we see that the sensitive volume drops by four orders of magnitude - from $526 \mathrm{Gpc}^{3}$ to $0.43 \mathrm{Gpc}^{3}$ - as we go from $q=1$ to $q \approx 60$. Even though the sub-dominant modes have doubled our sensitivity at the highest mass ratio tile, the magnitude of the sensitive volume is small relative to the other parts of the bank. Using the split bank is therefore unlikely to have a significant affect on the overall probability of making a gravitational-wave detection in advanced LIGO, unless there is a relatively large population of IMRIs with $q \gtrsim 4$ compared to IMBH-IMBH and stellar-mass BBHs.

\section{CONCLUSIONS AND FUTURE WORK}

We have investigated the effects of neglecting subdominant modes in templates on the predicted $\mathrm{BBH}$ sensitivity of a single advanced LIGO detector. In doing so we have considered the loss in re-weighted SNR, which more accurately reflects what is done in real $\mathrm{BBH}$ searches than considering SNR alone. We found that not including sub-dominant modes in templates does not make the sensitivity to non-spinning $\mathrm{BBH}$ signals any worse than what has been predicted as long as the gravitational waves can be detected at single-detector reweighted SNRs $\lesssim 9.5$. If the threshold for detection is larger, neglecting sub-dominant modes will result in worse sensitivity than predicted, but only for IMRI BBHs with total masses $\gtrsim 100 \mathrm{M}_{\odot}$ and $q \gtrsim 4$. Stellar-mass BBHs $\left(M \lesssim 100 \mathrm{M}_{\odot}\right)$ and more equal-mass IMBH-IMBH binaries $\left(m_{1,2} \gtrsim 100 \mathrm{M}_{\odot}, q \lesssim 4\right)$ are unaffected.

We have also simulated a bank of non-spinning templates that have sub-dominant modes to see if any improvement in sensitivity can be gained. To do so we analytically maximized the SNR over $\kappa$ when sub-dominant modes are included, then numerically maximized over the remaining extrinsic parameters [see Appendix A]. We found that if sub-dominant modes are used throughout the entire bank, and all templates are weighted equally, the sensitivity would be worse for stellar-mass BBHs and IMBH-IMBH binaries with $q \lesssim 4$ due to the increase in threshold needed to keep false-alarm probability fixed. Such a search would only improve sensitivity to IMRIs with $q \gtrsim 4$. Therefore, in order to include sub-dominant modes in templates, the templates must be weighted by the likelihood that they will detect a signal.

We found an effective weight by splitting the bank in two parts. Using sub-dominant modes in templates that satisfy Eq. 24) and dominant-mode templates elsewhere, we can improve the sensitivity to IMRI BBHs without sacrificing the sensitivity to stellar-mass BBHs and IMBH-IMBH binaries. The split bank is only one of many possible weighting schemes. Using sub-dominant modes throughout the entire bank with a more incremental weight may yield better sensitivity. However, since sub-dominant modes improve sensitivity to signals for which the detectors are least sensitive, a more sophisticated search would probably only yield a small increase in the detection rate.

In Fig. 10 we present the single-detector sensitive volumes of the split bank. We find that the sensitive volumes to signals in the sub-dominant part of the bank 


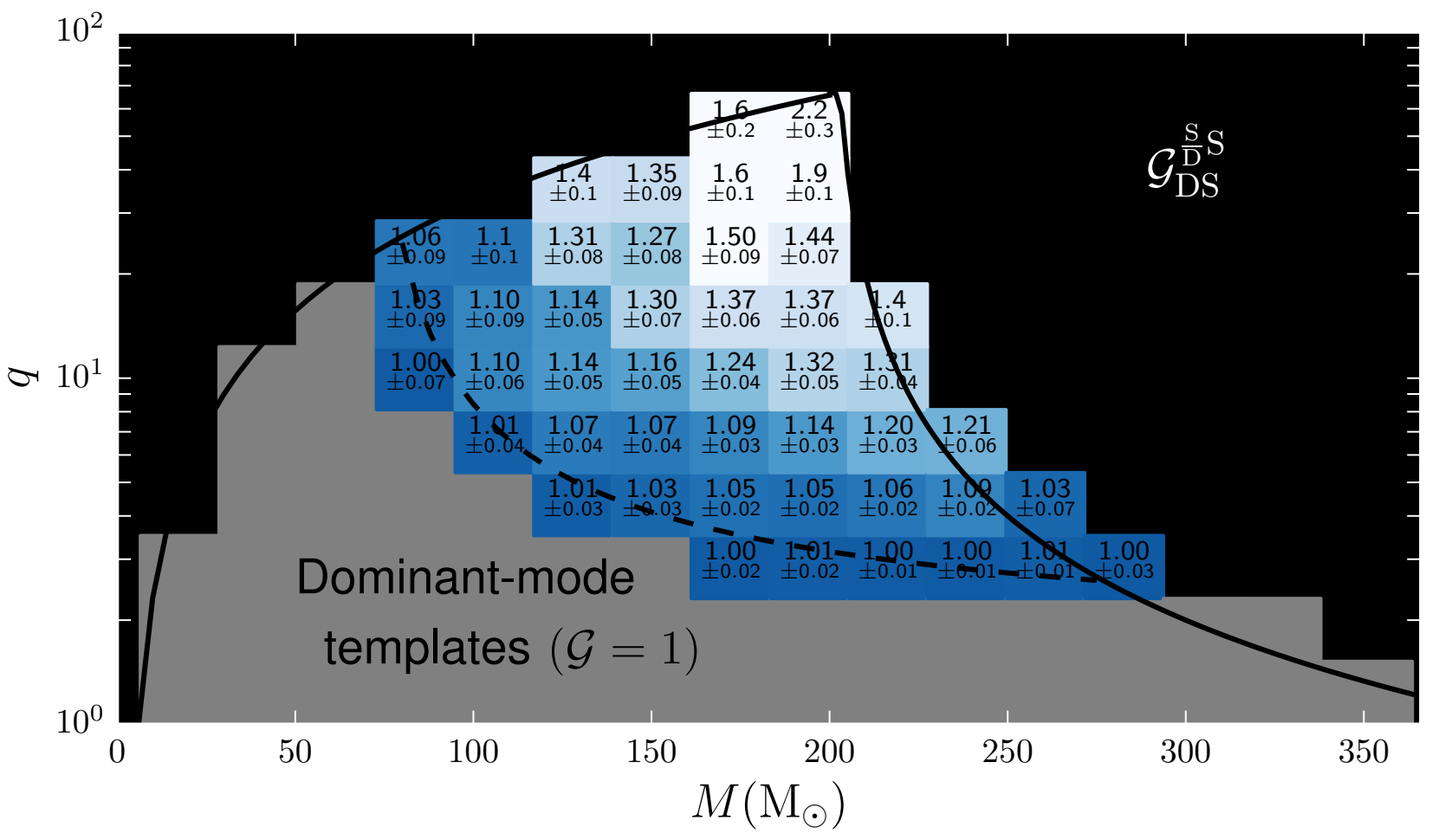

FIG. 9. Relative gain of sub-dominant bank compared to dominant-mode bank if the sub-dominant modes are only used in part of the bank, $\mathcal{G}_{\mathrm{DS}}^{\frac{\mathrm{S}}{\mathrm{D}} \mathrm{S}}$. The sold black line indicates the injection region; the dashed-black line indicates the boundary used for switching between dominant-mode and sub-dominant mode templates.

(tiles above the black-dashed line) are one to four orders of magnitude smaller than equal mass-ratio systems with equivalent total masses. Using the split-bank is therefore unlikely to substantially increase the probability of making a gravitational-wave detection over a dominant-mode bank unless there is a large population of high-mass ratio IMRIs in the universe.

In order to use a split bank more investigation is required to establish how exactly to use sub-dominant modes in a real search. Open questions include how to search over $\theta$ and $\phi{ }^{15}$ and how to apply a coincidence test between multiple detectors. Given that using a split bank has negligible impact on the overall probability of making a gravitational-wave detection in advanced LIGO, simply using a dominant-mode bank everywhere may be more desirable.

We did not try to predict advanced LIGO BBH detection rates, as doing so would require a choice of astrophysical rates. However, Fig. 10 can be used to predict detection rates if a particular astrophysical rate is assumed. The volumes given in Fig. 10 are for a split bank; dividing the sensitive volumes by the net gains

15 One possibility is to simply place templates in $\theta$ and $\phi$ using the stochastic method described in Ref. [53] In that case, the SNR of each template would be found using Eq. A20. given in Fig. 9yields the sensitive volumes if a dominantmode bank is used everywhere instead. For example, if a dominant-mode bank is used, the sensitive volume of the largest-mass ratio tile is $\sim 0.2 \mathrm{Gpc}^{3}$ instead of $0.43 \mathrm{Gpc}^{3}$. The sensitive volumes we report were calculated using a single detector. Since real searches use a network of detectors, actual sensitive volumes may vary depending on the relative sensitivities of each detector.

We emphasize that in this study we only considered non-spinning signals. Sub-dominant modes are likely to play a more important role when one or both of the component masses are spinning. Currently, there are no spinning waveform models available with merger and ringdown that include sub-dominant modes. Once such waveforms become available, creating a sub-dominant mode search may be more advantageous. Since our analytic maximization over $\kappa$ in Appendix A is still valid if the component masses are spinning, the result therein [specifically Eq. A20] can be used in such a search.

A dominant-mode EOB model calibrated to numerical relativity that incorporates spins aligned with the orbital angular momentum does currently exist [54], as well as spinning "phenomological" models derived from numerical relativity [55, 56. Past BBH searches have only used non-spinning templates, but there is much work currently on-going to extend template banks into the spinning regime [53, 57]. Doing so brings up many of the 


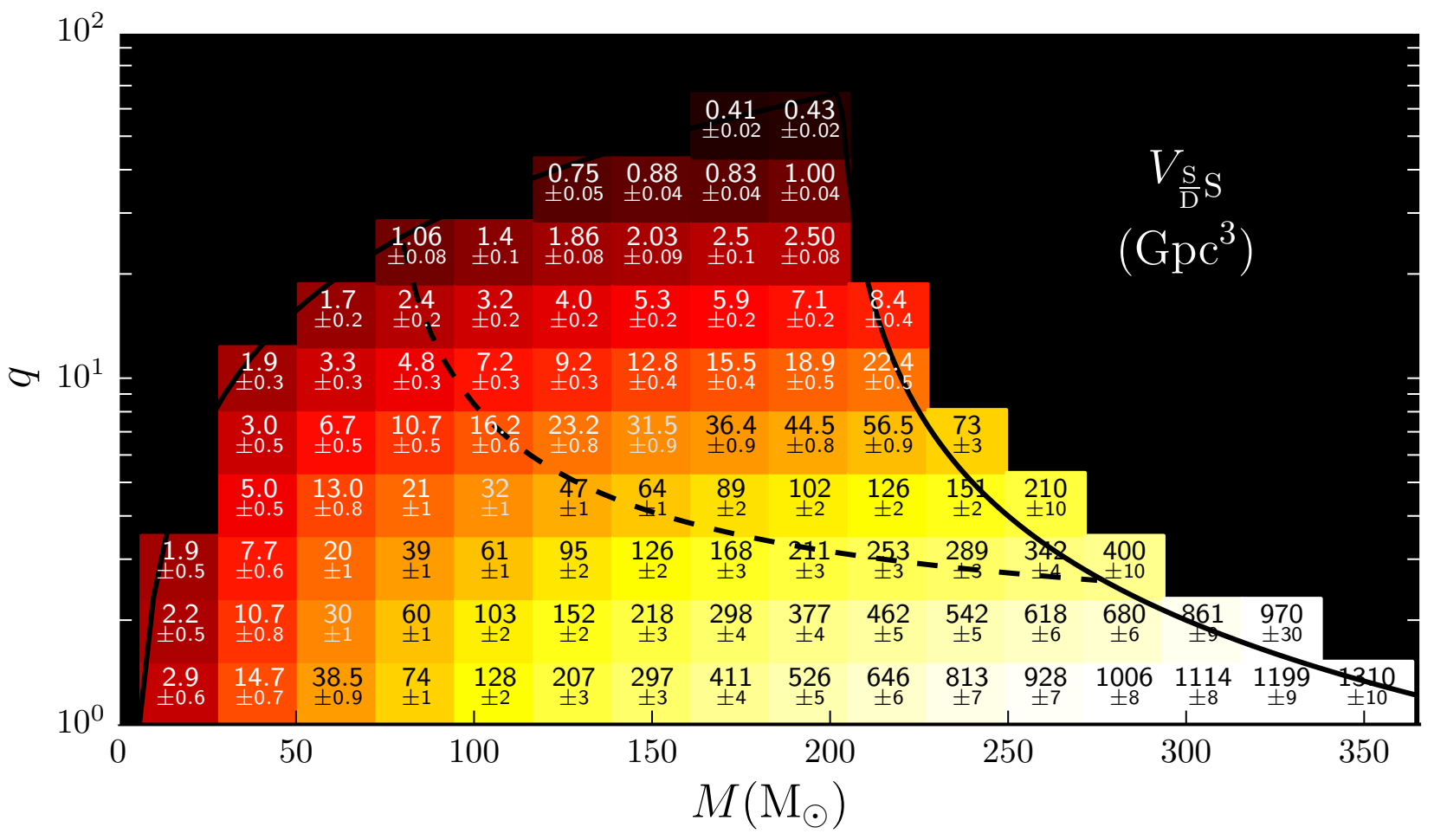

FIG. 10. Sensitive volume in $\mathrm{Gpc}^{3}$ of the split bank. The solid-black line shows the injection region, the dashed-black line the cut between sub-dominant and dominant-mode templates. Dividing the sensitive volumes in this figure by the net gains given in Fig. 9 yields sensitive volumes if dominant-mode templates are used everywhere.

same questions we addressed in this study: is the sensitivity of a search significantly affected by neglecting spin? Does the increase in number of templates needed to include spin increase the false-alarm probability such that any gain in SNR is nullified? In a future work we will address these questions using spinning EOB waveforms calibrated to numerical relativity and the methods that we establish in this study.

\section{ACKNOWLEDGMENTS}

We are grateful to Kipp Cannon, Thomas Dent, Prayush Kumar, Cole Miller, Alex Nitz, and John Whelan for valuable discussions. We would also like to thank Duncan Brown for providing some of the software used in this analysis. A.B., C.C. and Y.P. acknowledge partial support from NSF Grants No. PHY-0903631 and No. PHY-1208881. A.B. also acknowledges partial support from the NASA Grant NNX12AN10G. All calculations were performed on the SUGAR cluster, which is supported by NSF Grants No. PHY-1040231, No. PHY1104371, and No. PHY-0600953, and by Syracuse University ITS.

\section{Appendix A: SNR maximization with sub-dominant modes}

All of the assumptions we made for dominant-mode templates apply to templates with sub-dominant modes up to Eq. (8). As stated in Sec. II], when only the dominant-mode is considered, the angles $\kappa, \theta$, and $\phi$ can be combined into a single parameter. The maximization over this parameter is straight forward as the denominator of the SNR does not depend on it (the parameter cancels in the inner product $\sqrt{\langle h, h\rangle}$ ) [10]. However, when sub-dominant modes are considered, the maximization over the extrinsic parameters is more complicated. First, $\kappa, \theta$, and $\phi$ cannot be combined as the degeneracy between them is broken. Second, both the numerator and the denominator in the SNR depend on all three angles [see Eq. A19), below]. Here we derive an analytic expression for the SNR maximized over $\kappa$ when all modes are included in the waveform. As the result [Eq. A20] ] has non-trivial dependence on $\theta$ and $\phi$ we do the remaining maximizations numerically.

The $m$ summation in Eq. (2) is over $-l \leq m \leq l$. The number of terms in the summation can be reduced by relating the positive and negative $m$ modes. Letting:

$$
\begin{aligned}
{ }_{-2} Y_{l m}(\theta, \phi) & =g_{l m}(\theta) e^{i m \phi}, \\
h_{l m}(t) & =A_{l m}(t)^{-i m \Psi_{l m}(t)},
\end{aligned}
$$


$h$ is:

$$
\begin{aligned}
& h(t)=\sum_{l|m|}\left(\Re \left\{g_{l m}(\theta) e^{i m \phi} h_{l m}(t)\right.\right. \\
& \left.\left.+g_{l-m}(\theta) e^{-i m \phi} h_{l-m}(t)\right\}\right) \cos \kappa \\
& +\left(\Im \left\{g_{l m}(\theta) e^{i m \phi} h_{l m}(t)\right.\right. \\
& \left.\left.+g_{l-m}(\theta) e^{-i m \phi} h_{l-m}(t)\right\}\right) \sin \kappa .
\end{aligned}
$$

Here, $|m|$ indicates that the summation is over positive$m$ modes only, and we have set $\mathcal{D}=1$ since it does not enter in the SNR ${ }^{16}$ The $\pm m$ modes of the $h_{l m}$ are related by:

$$
h_{l-m}=(-1)^{l} h_{l m}^{*} .
$$

Using this relationship Eq. A1 becomes:

$$
\begin{aligned}
h(t)=\sum_{l|m|}\left(G_{1 l m}(\theta) \cos (m \phi) h_{1 l m}(t)\right. \\
\left.\quad+G_{1 l m}(\theta) \sin (m \phi) h_{2 l m}(t)\right) \cos \kappa \\
+\left(G_{2 l m}(\theta) \sin (m \phi) h_{1 l m}(t)\right. \\
\left.\quad-G_{2 l m} \cos (m \phi) h_{2 l m}(t)\right) \sin \kappa
\end{aligned}
$$

where:

$$
\begin{aligned}
G_{1 l m}(\theta) & =g_{l m}(\theta)+(-1)^{l} g_{l-m}(\theta) ; \\
G_{2 l m}(\theta) & =g_{l m}(\theta)-(-1)^{l} g_{l-m}(\theta) ; \\
h_{1 l m}(t) & =A_{l m}(t) \cos [m \Psi(t)] ; \\
h_{2 l m}(t) & =A_{l m}(t) \sin [m \Psi(t)] .
\end{aligned}
$$

Equation (A3) can be expressed more concisely by the matrix equation:

$$
\begin{aligned}
h(t) & =\sum_{l|m|} \mathbf{K} \mathcal{P}_{l m} \mathcal{H}_{l m} \\
& =\mathbf{K} \mathbf{P H},
\end{aligned}
$$

where:

$$
\begin{aligned}
& \mathcal{P}_{l m}=\left(\begin{array}{cc}
G_{1 l m} \cos m \phi & G_{1 l m} \sin m \phi \\
G_{2 l m} \sin m \phi & -G_{2 l m} \cos m \phi
\end{array}\right) ; \\
& \mathcal{H}_{l m}=\left(\begin{array}{l}
h_{1 l m} \\
h_{2 l m}
\end{array}\right) ; \\
& \mathbf{K}=\left[\begin{array}{ll}
\cos \kappa & \sin \kappa
\end{array}\right] \text {; } \\
& \mathbf{P}=\left[\begin{array}{lll}
\mathcal{P}_{22} & \mathcal{P}_{21} & \cdots
\end{array}\right] \\
& \mathbf{H}=\left[\begin{array}{c}
\mathcal{H}_{22} \\
\mathcal{H}_{21} \\
\vdots
\end{array}\right] \text {. }
\end{aligned}
$$

16 As written, Eq. A1 double counts terms for which $m=0$. For the sake of brevity we have redefined the $A_{l 0}$ in this equation to be $1 / 2$ of their original values.
Let us define the covariance matrix $\mathbf{C}$ as:

$$
\mathbf{C} \equiv \mathbf{H} \otimes \mathbf{H},
$$

where the outer product $\mathbf{A} \otimes \mathbf{B}$ is:

$$
[\mathbf{A} \otimes \mathbf{B}]_{i j} \equiv\left\langle A_{i}, B_{j}\right\rangle .
$$

Note that $\mathbf{K}$ depends only on $\kappa, \mathbf{P}$ on $\theta$ and $\phi$, and $\mathbf{C}$ on the intrinsic parameters $\mathbf{\Upsilon}$. Also note that $\mathbf{C}$ is symmetric. In this notation $\langle h, h\rangle$ is:

$$
\langle h, h\rangle=\mathbf{K P C P}^{\mathbf{T}} \mathbf{K}^{\mathbf{T}} .
$$

Defining $\mathbf{Q}$ as:

$$
\mathbf{Q}=\mathbf{H} \otimes[s]=\left[\begin{array}{c}
\left\langle h_{122}, s\right\rangle \\
\left\langle h_{222}, s\right\rangle \\
\vdots
\end{array}\right],
$$

the SNR is:

$$
\rho=\max _{\theta, \phi, \kappa} \frac{\mathbf{K P Q}}{\sqrt{\mathbf{K P C P}^{\mathbf{T}} \mathbf{K}^{\mathbf{T}}}} .
$$

We can perform the maximization over $\kappa$ analytically. First consider the case when $\theta=\pi / 2$. From the definition of the ${ }_{-2} Y_{l m}$ :

$$
G_{2 l m}(\pi / 2)=0, \forall(l, m),
$$

which means:

$$
P_{2 i}(\theta=\pi / 2, \phi)=0, \forall i .
$$

In this case $\mathbf{K P}$ has no $\sin \kappa$ terms, causing the $\kappa$ dependence to cancel in Eq. A19. Thus:

$$
\rho=\max _{\phi, \theta=\pi / 2} \frac{P_{1 i} Q_{i}}{\sqrt{P_{1 j} C_{j k} P_{k 1}}} .
$$

To maximize over $\kappa$ when $\theta \neq \pi / 2$, let $\mathbf{g}=\mathbf{P C P}^{\mathbf{T}}$ so that the denominator of the SNR is $\sqrt{\mathbf{K g K}^{\mathbf{T}}}$. Note that $\mathrm{g}$ is a $2 \times 2$ symmetric matrix. We can therefore think of it as a metric that defines an inner product space between 2-dimensional vectors. Since $\mathbf{g}$ depends on $\mathbf{P}$ and $\mathbf{C}$ the curvature of this space is determined by $\theta, \phi$ and $\Upsilon$. From this point of view the denominator of the SNR is simply the magnitude of $\mathbf{K}$ in this space 18

$$
\sqrt{\mathbf{K P C P}^{\mathbf{T}} \mathbf{K}^{\mathbf{T}}}=\sqrt{\mathbf{K g K}^{\mathbf{T}}} \equiv \sqrt{\langle\langle K, \mathbf{K}\rangle\rangle} \equiv\|\mathbf{K}\| .
$$

If we let $\mathbf{S}^{\mathbf{T}}=\mathbf{g}^{-1} \mathbf{P Q}$ then we find that the numerator of the SNR is the inner product of $\mathbf{K}$ and $\mathbf{S}$ :

$$
\mathbf{K P Q}=\mathbf{K g S}^{\mathbf{T}}=\langle\langle\mathbf{S}, \mathbf{K}\rangle\rangle .
$$

17 Here repeated indices indicate sum over.

18 We use $\langle\langle\cdot, \cdot\rangle\rangle$ to differentiate the inner product defined by $\mathbf{g}$ from the inner product defined in Eq. 4 . 
The SNR is therefore at a maximum when $\mathbf{K}$ and $\mathbf{S}$ are aligned:

$$
\rho=\max _{\theta, \phi, \kappa} \frac{\langle\langle\mathbf{S}, \mathbf{K}\rangle\rangle}{\|\mathbf{K}\|}=\max _{\theta, \phi}\|\mathbf{S}\| .
$$

The magnitude of $\mathbf{S}$ is:

$$
\|\mathbf{S}\|=\sqrt{\mathbf{S g S}^{\mathbf{T}}}=\sqrt{\mathbf{Q}^{\mathbf{T}} \mathbf{P}^{\mathbf{T}}\left(\mathbf{g}^{-1}\right)^{\mathbf{T}} \mathbf{g g}^{-1} \mathbf{P Q} .}
$$

Since $\mathbf{g}$ is symmetric its inverse is also symmetric. The SNR is thus:

$$
\rho= \begin{cases}\max _{\phi} P_{1 i} Q_{i}\left(P_{1 j} C_{j k} P_{k 1}\right)^{-1 / 2} & \text { if } \theta=\pi / 2, \\ \max _{\theta, \phi} \sqrt{\mathbf{Q}^{\mathbf{T}} \mathbf{P}^{\mathbf{T}}\left(\mathbf{P C P}^{\mathbf{T}}\right)^{-1} \mathbf{P Q}} & \text { otherwise. }\end{cases}
$$

Note that in performing the $\kappa$ maximization we did not need to invoke the non-spinning assumption. Thus the argument of Eq. A20 is valid for all systems; if there is spin, then further maximizations would need to be carried out over the spin components in addition to $\theta$ and $\phi$.

\section{Appendix B: Estimating false-alarm probability from a bank of templates}

The false-alarm probability of a template bank as a function of SNR depends on the size of the parameter space being searched over. The larger the parameter space, the greater the probability of getting a false alarm. Let us assume that every template in the bank is independent of each other. As described in Sec. III in a search, intrinsic parameters are maximized over by selecting the template that yields the largest SNR. Due to this maximization, only one template can produce a trigger at any given time, making the probability of getting a trigger with some SNR from each template mutually exclusive. The false-alarm probability of the bank is thus:

$$
\mathcal{F}(\rho)=\sum_{k=1}^{N} \int_{\rho}^{\infty} f_{B}\left(\rho^{\prime} \mid k\right) \mathrm{d} \rho^{\prime},
$$

where $N$ is the number of templates in the bank and $f_{B}\left(\rho^{\prime} \mid k\right)$ is the probability density function of the SNR of each template after maximization. Due to the bank maximization $f_{B}(\rho \mid k)$ is given by the probability that the template produces an SNR equal to $\rho$ times the probability that every other template produces an SNR less than $\rho$; thus:

$$
\begin{aligned}
\mathcal{F}(\rho) & =\sum_{k=1}^{N} \int_{\rho}^{\infty} f_{P}\left(\rho^{\prime} \mid k\right) \prod_{l \neq k}^{N}\left[\int_{0}^{\rho^{\prime}} f_{P}\left(\rho^{\prime \prime} \mid l\right) \mathrm{d} \rho^{\prime \prime}\right] \mathrm{d} \rho^{\prime} \\
& =\sum_{i=1}^{N} \int_{\rho}^{\infty} f_{P}\left(\rho^{\prime} \mid k\right) \prod_{l \neq k}^{N} F_{P}\left(\rho^{\prime} \mid l\right) \mathrm{d} \rho^{\prime} .
\end{aligned}
$$

Here $f_{P}(\rho \mid k)$ is the SNR distribution in noise of the $k^{\text {th }}$ template and $F_{P}(\rho \mid l)$ is the cumulative distribution function of the $l^{\text {th }}$ template.

In Gaussian noise, every dominant-mode template is $\chi$ distributed with two degrees of freedom. For a dominantmode bank Eq. (B1) simplifies to:

$$
\mathcal{F}_{\mathrm{D}}(\rho)=1-\left(1-e^{-\frac{\rho^{2}}{2}}\right)^{N} .
$$

In our case, $N=19800$ and $\rho=8$; the probability that the dominant-mode bank produces a false alarm at SNR 8 is therefore $\approx 2.5 \times 10^{-10}$.

Note that Eq. (B2) approaches 1 as $N \rightarrow \infty$ for all $\rho>0$. This is due to our assumption that the templates are independent of each other. We expect that as we increase the minimal match of the bank to $100 \%$ (which would require an infinite number of templates) the false-alarm probability would instead approach some limiting value for a given $\rho$. The minimal match of our template bank is $97 \%$; assuming that templates are independent might therefore seem exceedingly naïve. To test this assumption we filtered the dominant mode template bank in 10000 realizations of noise. In each realization we selected the template with the best SNR to provide a measure of false-alarm probability as a function of SNR. We found good agreement between these results and Eq. (B2). Thus, despite a seemingly large overlap between neighboring templates, our assumption of independence appears to be approximately valid for the minimal match of our bank.

To model a search with sub-dominant modes we assume a bank with the same number of templates and with the same $(M, q)$ coordinates as the dominant-mode bank. In such a search we would maximize $\rho$ over $\theta, \phi$ and $\kappa$ for each template, then maximize over the entire bank. To find $\mathcal{F}(\rho)$ of this bank we need the probability density function of the SNR for each template in stationary Gaussian noise, $f_{P}(\rho \mid k)$. To find that we need an expression for the maximized SNR when sub-dominant modes are included. In Appendix A we analytically maximize $\rho$ over $\kappa$ to get Eq. A20). This equation depends on the matrix $\mathbf{Q}$, the elements of which are the overlap between the template and the detector data [see Eq. [A18]]. In stationary Gaussian noise the $Q_{i}$ are Gaussian random variables with variance $\sigma_{i}^{2}=\sum_{j} C_{i j}$, where $C_{i j}$ are the elements of the covariance matrix defined in Eq. (A15). To find $f_{P}(\rho \mid k)$ from this multivariate Gaussian distribution we need to maximize over $\theta$ and $\phi$. This maximization is not trivial, however, and so we must find the SNR distribution numerically.

One way to find $f_{P}(\rho \mid k)$ is to generate many realizations of noise, filter it to get $\mathbf{Q}$, then perform the maximization in Eq. A20 for each template. However, we expect the probability of getting $\rho \approx 8$ to be extremely small: the probability of getting $8 \pm 0.1$ from a $\chi$ distribution with 10 degrees of freedom (the upper limit of what we expect from a template with 5 modes) is order $10^{-9}$. Getting an accurate measure of $f_{P}(\rho \mid k)$ around SNR 8 is 
thus computationally intractable using this "brute force" method. Instead, we follow a procedure similar to that used in Ref. [58 to find the probability density function.

To simulate a particular realization of $\mathbf{Q}$ we do not need to do any matched filtering; instead we draw a set $\mathbf{Z}$ of pseudo-random values from a Gaussian distribution with zero mean and unit variance. $\mathbf{Q}$ is ther 19 ,

$$
Q_{i}=\sqrt{C_{i j}} Z_{j} .
$$

We think of $\mathbf{Q}$ as being a vector in a $\nu$ dimensional space $\mathcal{S}$, where $\nu$ is equal to twice the number of modes; the relative size of each dimension is determined by the covariance matrix.

Now let $r=\|\mathbf{Z}\|$ and $\|\hat{\mathbf{Z}}\|=1$ such that $\mathbf{Z}=r \hat{\mathbf{Z}}$. Define $\hat{\rho}$ to be the maximized SNR we obtain from a realization of $\hat{\mathbf{Q}}=\sqrt{\mathbf{C}} \hat{\mathbf{Z}}$. From From Eq. A20 we see that:

$$
\rho(\mathbf{Q}, k)=r \hat{\rho}(\hat{\mathbf{Q}}, k) \equiv r \hat{\rho}(\Omega, k) .
$$

Here, $\Omega$ is the solid angle describing the direction of $\mathbf{Q}$ in $\mathcal{S}$, and we have made the dependence of the SNR on the intrinsic parameters of the $k^{\text {th }}$ template explicit. Since each element in $\mathbf{Z}$ is a Gaussian random variable with zero mean and unit variance, $r$ - which is the quadrature sum of these variables - is $\chi$ distributed with $\nu$ degrees of freedom:

$$
f_{R}(r \mid \nu)=\frac{r^{\nu-1} e^{-r^{2} / 2}}{2^{\nu / 2-1} \Gamma(\nu / 2)} .
$$

The number of degrees of freedom is equal to the dimension of $\mathbf{Q}$, which is twice the number of modes. In our case, $\nu=10$.

Using the coordinate transformation given by Eq. (B3) we find:

$$
f_{P}(\rho \mid k, \Omega, \nu)=\frac{1}{\hat{\rho}(\Omega, k)} f_{R}\left(\frac{\rho}{\hat{\rho}(\Omega, k)} \mid \nu\right) .
$$

Marginalizing out $\Omega$ yields $f_{P}(\rho \mid k, \nu)$ :

$$
f_{P}(\rho \mid k, \nu)=\int \frac{1}{\hat{\rho}(\Omega, k)} f_{R}\left(\frac{\rho}{\hat{\rho}(\Omega, k)} \mid \nu\right) \mathrm{d} \Omega / \int \mathrm{d} \Omega .
$$

We solve this via Monte Carlo integration. For each point in the Monte Carlo we generate a normalized random vector $\hat{\mathbf{Z}}$. We use Eq. A20 to find $\hat{\rho}(\Omega)$ for this realization, with $\hat{\mathbf{Q}}=\sqrt{\mathbf{C}} \hat{\mathbf{Z}}$. We then find $f_{P}(\rho \mid k, \nu)$ with $\nu=10$ for several different values of $\rho$, terminating the Monte Carlo when the error on $f_{P}(\rho \mid k)$ at $\rho=9$ is less than $20 \%$.

The top plot in Fig. 11 shows $f_{P}(\rho \mid k)$ for two different templates. We find that for $\rho \gtrsim 7, f_{P}(\rho \mid k)$ approaches

19 Since $\mathbf{C}$ is positive-definite $\sqrt{\mathbf{C}}$ is real; we find it from the eigendecomposition of $\mathbf{C}$. Specifically, $\sqrt{\mathbf{C}}=\mathbf{T} \sqrt{\boldsymbol{\Lambda}} \mathbf{T}^{-1}$ where $\mathbf{T}$ is the matrix of the eigenvectors of $\mathbf{C}$ and $\boldsymbol{\Lambda}$ is the diagonal matrix formed from the eigenvalues.
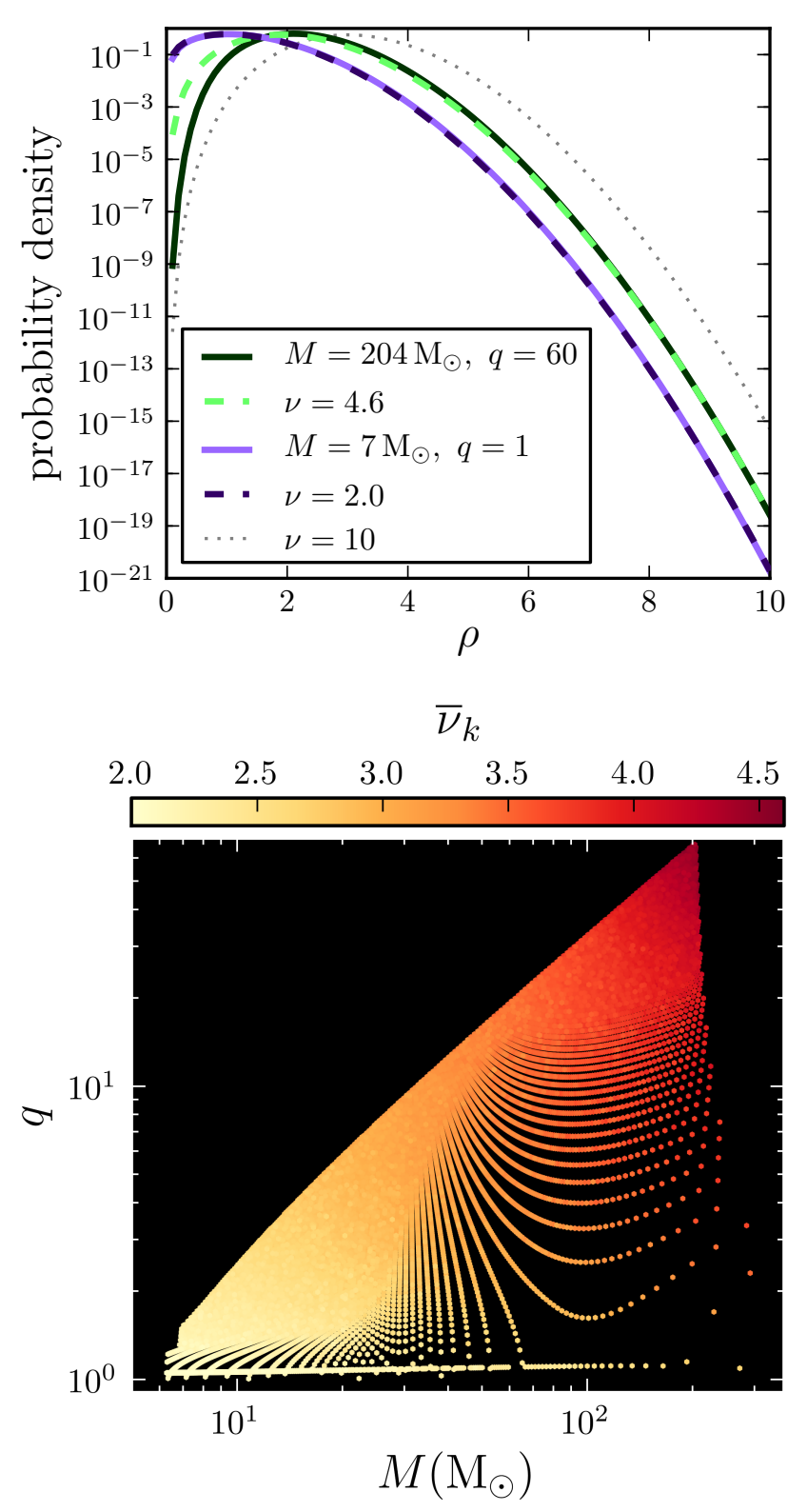

FIG. 11. SNR distribution in noise of two templates with sub-dominant modes $\left(f_{P}(\rho \mid k)\right)$ versus SNR (top) and fitted number of degrees of freedom $\bar{\nu}_{k}$ for each template in total mass and mass-ratio $q$ (bottom). The dashed lines in the top plot show a $\chi$ distribution with the fitted $\bar{\nu}_{k}$ to $f_{P}(\rho \mid k)$ of the templates shown. Fits were done between $\rho=7$ and 9 .

the $\chi$ distribution with non-integer number of degrees of freedom $f_{X}\left(\rho \mid \nu_{k}\right)$ for all templates. We therefore fit $f_{X}\left(\rho \mid \nu_{k}\right)$ to $f_{P}(\rho \mid k)$ between $\rho=7$ and 9 by maximizing over $\nu_{k}$. The bottom plot in Figure 11, shows the best-fit $\nu_{k}\left(\bar{\nu}_{k}\right)$ for each template in the bank. If all of the modes were independent of each other $\bar{\nu}_{k}$ would be equal to 10 for all $k$. Instead we find that the largest $\bar{\nu}_{k} \approx 4.6$, which occurs at the largest mass-ratio and total mass part of the bank. As the templates approach the equal mass line the number of degrees of freedom approaches two. This 
is expected: as $q \rightarrow 1$, the sub-dominant modes become small relative to the dominant mode, and $f_{P}(\rho \mid k)$ reduces to a $\chi$ distribution with 2 degrees of freedom.

To solve Eq. B1 we substitute $f_{X}\left(\rho \mid \bar{\nu}_{k}\right)$ and
$F_{X}\left(\rho \mid \bar{\nu}_{k}\right)$ for $f_{P}(\rho \mid k)$ and $F_{P}(\rho \mid k)$, then numerically integrate for several different values of $\rho$. Inverting yields $\rho(\mathcal{F})$, which we solve for $\mathcal{F}=\mathcal{F}_{\mathrm{D}}(\rho=8)$.
[1] G. M. Harry and the LIGO Scientific Collaboration, Class. Quantum Grav. 27, 084006 (2010).

[2] T. Accadia et al. (Virgo Collaboration), "Advanced Virgo technical design report," https://tds.ego-gw.it/itf/ tds/file.php?callFile=VIR-0128A-12.pdf (2012).

[3] K. Somiya (KAGRA Collaboration), Class. Quantum Grav. 29, 124007 (2012), arXiv:1111.7185 [gr-qc]

[4] "IndiGO," http://www.gw-indigo.org/tiki-index. $\mathrm{php}$.

[5] J. Abadie et al. (LIGO Scientific Collaboration, Virgo Collaboration), Class. Quantum Grav. 27, 173001 (2010).

[6] J. Abadie et al. (LIGO Collaboration, Virgo Collaboration), Phys. Rev. D85, 082002 (2012), arXiv:1111.7314 $[\mathrm{gr}-\mathrm{qc}]$.

[7] J. Aasi et al. (LIGO Scientific Collaboration, Virgo Collaboration), (2012), arXiv:1209.6533 [gr-qc],

[8] J. Abadie et al. (The LIGO Scientific Collaboration and The Virgo Collaboration), Phys. Rev. D 85, 122007 (2012)

[9] C. Pankow, S. Klimenko, G. Mitselmakher, I. Yakushin, G. Vedovato, M. Drago, R. A. Mercer, and P. Ajith, Class. Quantum Grav. 26, 204004 (2009).

[10] B. Sathyaprakash and S. Dhurandhar, Phys. Rev. D44, 3819 (1991).

[11] R. Balasubramanian, B. S. Sathyaprakash, and S. V. Dhurandhar, Phys. Rev. D 53, 3033 (1996), arXiv:grqc/9508011

[12] B. J. Owen, Phys. Rev. D 53, 6749 (1996).

[13] Y. Pan, A. Buonanno, M. Boyle, L. T. Buchman, L. E. Kidder, et al., Phys. Rev. D84, 124052 (2011), arXiv:1106.1021 [gr-qc].

[14] L. Pekowsky, J. Healy, D. Shoemaker, and P. Laguna, (2012), arXiv:1210.1891 [gr-qc]

[15] D. A. Brown, P. Kumar, and A. H. Nitz, Phys. Rev. D 87, 082004 (2013), arXiv:1211.6184 [gr-qc]

[16] D. McKechan, On the use of higher order waveforms in the search for gravitational waves emitted by compact binary coalescences, Ph.D. thesis, Cardiff University (2011), arXiv:1102.1749 [gr-qc]

[17] A. Buonanno and T. Damour, Phys. Rev. D59, 084006 (1999), arXiv:gr-qc/9811091 [gr-qc].

[18] A. Buonanno and T. Damour, Phys. Rev. D62, 064015 (2000), arXiv:gr-qc/0001013 [gr-qc]

[19] A. Buonanno, Y. Pan, J. G. Baker, J. Centrella, B. J. Kelly, et al., Phys. Rev. D76, 104049 (2007).

[20] T. Damour, A. Nagar, and S. Bernuzzi, (2012), arXiv:1212.4357 [gr-qc]

[21] T. B. Littenberg, J. G. Baker, A. Buonanno, and B. J. Kelly, Phys. Rev. D 87, 104003 (2012), arXiv:1210.0893 [gr-qc],

[22] L. T. Buchman, H. P. Pfeiffer, M. A. Scheel, and B. Szilagyi, Phys. Rev. D86, 084033 (2012), arXiv:1206.3015 [gr-qc],

[23] Y. Pan et al., (2013), in preparation.
[24] I. Hinder et al. (The NRAR Collaboration, Perimeter Institute for Theoretical Physics), (2013), arXiv:1307.5307 [gr-qc]

[25] B. Allen, Phys. Rev. D 71, 062001 (2005).

[26] S. Babak et al., Phys. Rev. D 87, 024033 (2013), arXiv:1208.3491 [gr-qc].

[27] J. Abadie et al. (LIGO Scientific Collaboration and Virgo Collaboration), Phys. Rev. D 83, 122005 (2011).

[28] M. C. Miller and E. J. M. Colbert, International Journal of Modern Physics D 13, 1 (2004) astro-ph/0308402.

[29] D. Shoemaker (LIGO Collaboration), "Advanced LIGO anticipated sensitivity curves," (2010), LIGO Document T0900288-v3.

[30] J. Aasi et al. (VIRGO Collaboration), Class.Quant.Grav. 29, 155002 (2012), arXiv:1203.5613 [gr-qc]

[31] J. McIver (for the LIGO Sceintific Collaboration and the Virgo Collaboration), Class. Quantum Grav. 29, 124010 (2012)

[32] J. Aasi et al. (the LIGO Scientific Collaboration, the Virgo Collaboration), (2013), arXiv:1304.1775 [gr-qc]

[33] L. S. Finn and D. F. Chernoff, Phys. Rev. D 47, 2198 (1993), arXiv:gr-qc/9301003

[34] B. F. Schutz and M. Tinto, Mon. Not. Roy. Astr. Soc. 224, 131 (1987).

[35] B. Allen, W. G. Anderson, P. R. Brady, D. A. Brown, and J. D. E. Creighton, Phys. Rev. D 85, 122006 (2012).

[36] T. Apostolatos, Phys. Rev. D52, 605 (1995).

[37] T. Damour, B. R. Iyer, and B. Sathyaprakash, Phys. Rev. D57, 885 (1998), arXiv:gr-qc/9708034 [gr-qc]

[38] P. R. Brady, J. D. E. Creighton, and A. G. Wiseman, Class. Quantum Grav. 21, S1775 (2004).

[39] A. Agresti and B. A. Coull, The American Statistician 52, 119 (1998).

[40] "The 2012-2013 data analysis, software and computing, detector characterization white paper," (2012), IIGO Document T1200286-v3.

[41] J. M. Silverman and A. V. Filippenko, The Astrophysical Journal 678, L17 (2008).

[42] P. A. Crowther, R. Barnard, S. Carpano, J. S. Clark, V. S. Dhillon, and A. M. T. Pollock, Mon. Not. Roy. Astron. Soc. , L11+ (2010), arXiv:1001.4616.

[43] K. Belczynski et al., Astrophys. J. 714, 1217 (2010), arXiv:0904.2784 [astro-ph.SR]

[44] M. Dominik, K. Belczynski, C. Fryer, D. Holz, E. Berti, T. Bulik, I. Mandel, and R. O'Shaughnessy, Astrophys. J. 759, 52 (2012), arXiv:1202.4901 [astro-ph.HE].

[45] M. Dominik, K. Belczynski, C. Fryer, D. E. Holz, E. Berti, T. Bulik, I. Mandel, and R. O'Shaughnessy, ArXiv e-prints (2013), arXiv:1308.1546v2 [astro-ph.HE]

[46] M. C. Miller, Class. Quant. Grav 26, 094031 (2009) arXiv:0812.3028

[47] D. A. Brown, J. Brink, H. Fang, J. R. Gair, C. Li, G. Lovelace, I. Mandel, and K. S. Thorne, Phys. Rev. Lett. 99, 201102 (2007), arXiv:gr-qc/0612060.

[48] "LSC Algorithm Library Suite," http://www. 
lsc-group.phys.uwm .edu/lal

[49] T. Cokelaer, Phys. Rev. D 76, 102004 (2007), arXiv:0706.4437.

[50] B. J. Owen and B. S. Sathyaprakash, Phys. Rev. D 60, 022002 (1999).

[51] S. Babak, R. Balasubramanian, D. Churches, T. Cokelaer, and B. S. Sathyaprakash, Class. Quant. Grav. 23, 5477 (2006), gr-qc/0604037

[52] D. Keppel, A. P. Lundgren, B. J. Owen, and H. Zhu, ArXiv e-prints (2013), arXiv:1305.5381 [gr-qc]

[53] P. Ajith, N. Fotopoulos, S. Privitera, A. Neunzert, and A. J. Weinstein, ArXiv e-prints (2012), arXiv:1210.6666 $[\mathrm{gr}-\mathrm{qc}]$

[54] A. Taracchini, Y. Pan, A. Buonanno, E. Barausse, M. Boyle, T. Chu, G. Lovelace, H. P. Pfeiffer, and M. A. Scheel, Phys. Rev. D 86, 024011 (2012).

[55] P. Ajith et al., Phys. Rev. Lett. 106, 241101 (2011), arXiv:arXiv:0909.2867 [gr-qc]

[56] L. Santamaria et al., Phys. Rev. D 82, 064016 (2010), arXiv:1005.3306 [gr-qc].

[57] D. A. Brown, I. Harry, A. Lundgren, and A. H. Nietz, Phys. Rev. D 86, 084017 (2012).

[58] Y. Pan, A. Buonanno, Y. Chen, and M. Vallisneri, Phys. Rev. D69, 104017 (2004). 